\title{
ESPACIALIDADES CHULLPARIAS. APROXIMACIÓN A LOS ANCESTROS desde la materialidad (Coranzulí, Jujuy, Argentina)
}

\author{
María Carolina Rivet ${ }^{1}$
}

\section{$*$ Introducción}

Resumen

En este artículo se propone abordar un análisis sobre los agrupamientos de estructuras chullparias, del área de Coranzulí, en las tierras altas de la provincia de Jujuy (Argentina), para el período de Desarrollos Regionales. Se expone un análisis de las características constructivas, de los fechados radiocarbónicos realizados y las configuraciones espaciales de estos agrupamientos. Estas líneas de evidencia, junto con distintas referencias arqueológicas, etnohistóricas y etnográficas para diversos lugares en los Andes permitirán reflexionar sobre los vínculos entre las personas y los ancestros y cómo la materialidad es clave para su constitución y sostenimiento en el tiempo.

Palabras claves: agrupamientos chullparios - espacialidades - ancestros Puna de Jujuy.

\begin{abstract}
This paper presents a study of clusters of chullparian structures in the area of Coranzulí, located in the highlands of Jujuy (Argentina) from the Regional Development period. An analysis of the construction characteristics, radiocarbon dates, and spatial configurations of these clusters is presented. These lines of evidence, along with other archaeological, ethnohistorical and ethnographic references to various places in the Andes, will allow for a reflection on the links between people and their ancestors, and how materiality is key for its establishment and maintenance in time.
\end{abstract}

Abstract

Key words: chullparian clusters - spatialities - ancestors - Puna de Jujuy.

Recibido: agosto 2014. Aceptado: Febrero 2015
Una de las características que parecieran definir a las estructuras chullparias en los Andes es que tienden a presentarse formando parte de agrupamientos (Isbell 1997; Lau 2008). En términos generales, éstos tienden a conformarse a partir de construcciones individuales a diferentes distancias entre sí, es decir, que no se encuentran adosadas unas a otras (Aldunate y Castro 1981; Gisbert et al. 1994; Kesseli y Pärssinen 2005; Tantaleán 2006, entre otros). En lo que respecta a la Puna de la provincia de Jujuy, los casos descriptos presentan la particularidad de que las estructuras muchas veces se integran entre sí, no solo por proximidad, sino que también presentan vínculos físicos concretos. Tal como se desarrollará, estos vínculos pueden implicar contactos explícitos entre los muros de las estructuras, a tal punto que pueden llegar a ser compartidos. Es así como, tanto en el caso del río San Juan Mayo como en el área de Casabindo y Doncellas, las estructuras suelen presentarse articuladas entre sí de tal manera que llegan incluso a compartir algunos de sus muros (Debenedetti 1930; Casanova 1938; Vignati 1938; Krapovickas 1958-59; Krapovickas y Cigliano 1962; Albeck y Ruiz 2003). Aunque es sumamente frecuente en esta área, esta situación también ha sido observada en otras, como por ejemplo en Lípez, particularmente en el sitio Llacta Khaka (Nielsen 2010). Esta es el tipo de configuración espacial que, precisamente, presentan los agrupamientos chullparios que hemos estudiado en el área de Coranzulí, en la Puna de Jujuy (noroeste de Argentina).

La conformación de los agrupamientos y el tipo de vínculos que se registra entre estructuras en los tres sitios que hemos trabajado se constituyen como un problema en sí

1 CONICET, Instituto Interdisciplinario Tilcara. Facultad de Filosofía y Letras, Universidad de Buenos Aires. Belgrano 445-Tilcara, Jujuy, ARGENTINA. Email: carolinarivet@hotmail.com 
mismo que, al recorrerlo, nos enfrenta a dos cuestiones de importancia para este artículo. Por un lado, las posibilidades que brinda el estudio de la materialidad (sensu Miller 2005) y de las decisiones técnicas para reflexionar sobre distintos aspectos de la vida social de las poblaciones andinas. Por el otro, y articulado a lo anterior, el rol de los ancestros en estas sociedades durante el período de Desarrollos Regionales, y el tipo de relaciones que se establecían entre éstos y las personas. La trascendencia de los ancestros para estas poblaciones ha sido ampliamente referida en términos arqueológicos, etnohistóricos y etnográficos para distintos lugares de los Andes (p.e. Zuidema 1973; Duviols 1979; Kaulicke 2001; Nielsen 2006; Lau 2008), y lo propio ocurre respecto a la asociación de éstos con las estructuras chullparias (p.e. Isbell 1997; Nielsen 2008; Gil García 2010; Duchesne y Chacama 2012). Lo que nos interesa aquí es concentrarnos en el estudio mismo de la arquitectura, y construcción de estas estructuras, como un camino posible y productivo para indagar sobre la ancestralidad. Más que preguntarnos sobre la estructura misma, lo que nos proponemos es un cambio de escala en el análisis para reconocer cómo éstas se integran entre sí.

Las configuraciones arquitectónicas y espaciales participan activamente en la definición de las conformaciones sociales, sin que esto implique que las determine. En relación con las estructuras chullparias, esto es importante por al menos dos motivos. En primer lugar, si estamos aceptando la existencia de una asociación entre chullpas y ancestros, las resoluciones técnicas que se seleccionan, en términos de morfologías, materiales y terminaciones, podrían estar produciendo y reforzando los conceptos que definen a estos ancestros como tales, y condicionando de una manera específica el tipo de relaciones con sus descendientes. Esto implica partir de la comprensión de que las decisiones técnicas, en tanto respuestas a un problema, están culturalmente definidas y, entonces, no pueden ser disociadas de otras prácticas (Lemonnier 1992, Sillar 1996, Dietler y Herbich 1998, entre otros). En segundo lugar, a partir del modo en que las estructuras se organizan en los agrupamientos, se podría haber establecido un ordenamiento a través del cual se producían y reproducían las relaciones entre los ancestros. Como ha planteado Nielsen (1995), la posición de una determinada estructura arquitectónica no equivale directamente a una posición social en términos jerárquicos, pero sí puede contribuir a reforzar el lugar de las personas, y en este caso también de los ancestros, dentro de la estructura social.

En concreto, en este artículo caracterizaremos los distintos tipos de agrupamientos chullparios que hemos registrado en Coranzulí, como paso previo para el análisis en detalle de tres casos específicos. Esto nos permitirá indagar en los agrupamientos como una construcción en el tiempo, a través de la propuesta de secuencias constructivas, y observar las particularidades de las configuraciones espaciales resultantes. $\mathrm{Al}$ articular este estudio con referencias etnohistóricas y etnográficas, podremos proponer un paralelo entre estas relaciones espaciales y los vínculos con los ancestros.

\section{El área de estudio}

A modo de aproximación general, el pueblo de Coranzulí ( $23^{\circ} 00^{\prime} 49^{\prime \prime}$ S, 66 $21^{\prime} 59^{\prime \prime}$ ) está ubicado dentro del departamento de Susques, en su extremo noreste, en la provincia de Jujuy, a unos $95 \mathrm{~km}$ al sudoeste de la localidad de Abra Pampa, a través de la Ruta Provincial N 74 , y a unos $70 \mathrm{~km}$ al noreste de Susques, cabecera del departamento, a partir de la nueva traza de la Ruta Nacional $\mathrm{N}^{\circ} 40$ (Figura 1). Toda el área está enmarcada hacia el este por la Sierra de Incahuasi y hacia el norte por la de Quichagua, siendo que el poblado, y los sitios arqueológicos, se emplazan al pie del cerro Coranzulí o Moraya, que posee una altura por encima de los $5000 \mathrm{msnm}$. Buena parte de nuestra área de trabajo se encuentra recorrida por el río Coranzulí, de agua permanente, que recibe aportes de distintos cauces menores, y a su vez alimenta, hacia el sur, al río de las Burras.

En términos topográficos y ambientales, Coranzulí se emplaza dentro de lo que se conoce como la Puna, es decir, una planicie de altura semidesértica, cuya altura oscila entre los $3000 \mathrm{msnm}$ y por encima de los 4000 msnm. De acuerdo a Cabrera (1953), esta área corresponde a la Provincia Fitogeográfica Puneña, con vegetación de los siguientes tipos: estepa arbustiva, estepa herbácea, estepa halófila, estepa sammófila y vegas. En general presenta un clima frío y seco, con una gran amplitud térmica diaria, y lluvias casi exclusivamente en época estival, que disminuyen de norte a sur, siendo muy escasas en su porción meridional. Coranzulí formaría parte de lo que se 


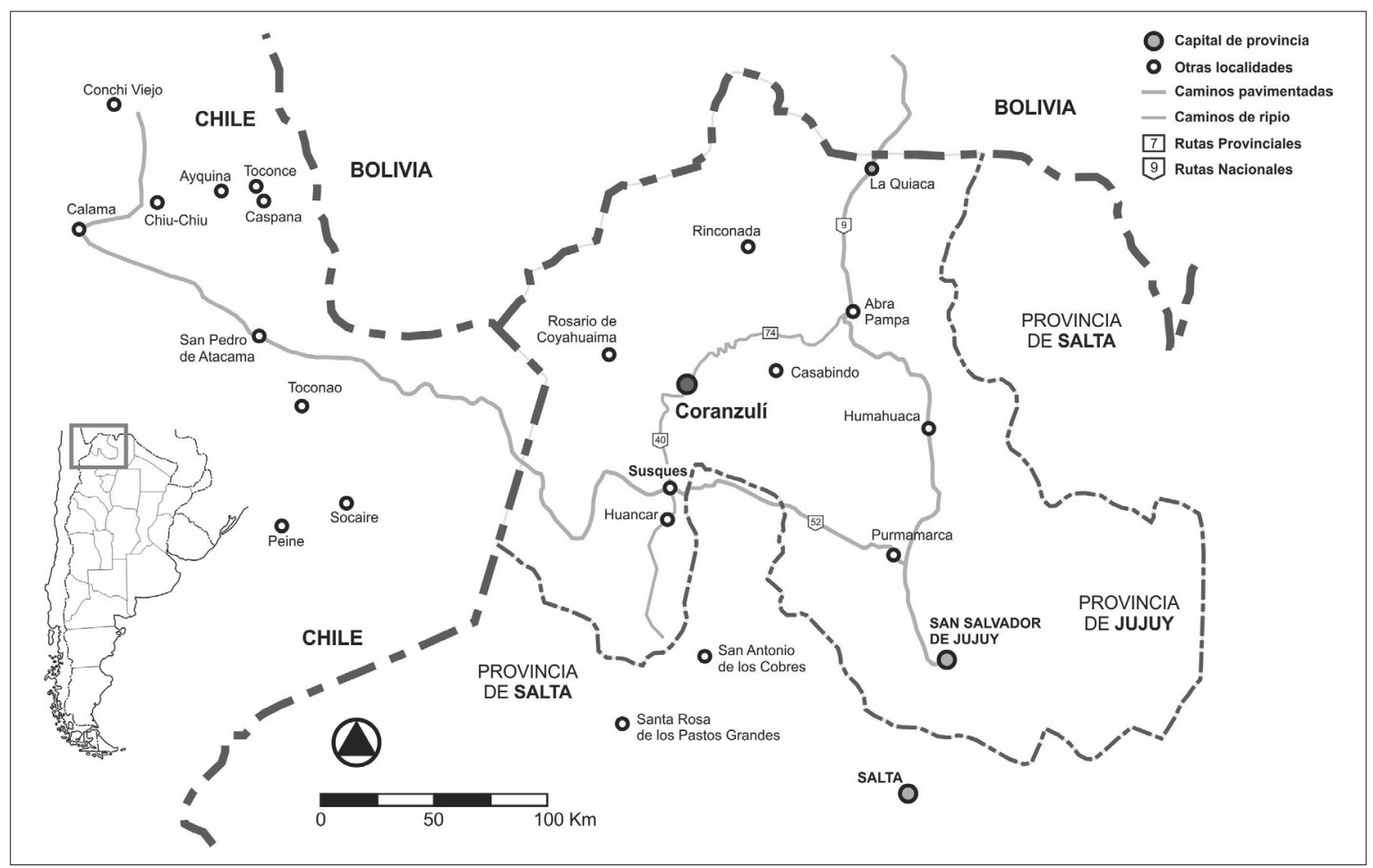

Figura 1. Mapa de ubicación de Coranzulí, en la provincia de Jujuy.

conoce como Puna Seca (Cabrera 1953) que presenta una mayor humedad, con formación de lagunas, ríos de agua permanente y una vegetación relativamente continua.

El trabajo que venimos desarrollando en los últimos años se concentró particularmente en tres sitios, dentro de lo que localmente se conoce como Sección Agua Delgada: Canalita (CAN), Wayra Wasi (WAY) y Licante (LIC). Estos tres sitios se caracterizan por la presencia de una gran cantidad de estructuras chullparias (101 unidades). Los tres sitios se emplazan en quebradas angostas delimitadas por farallones de ignimbritas. Para el caso de Licante, especialmente, hemos registrado la existencia de un área con recintos habitacionales, y sectores con infraestructura agrícola, particularmente terrazas de cultivo. En términos generales, las estructuras chullparias en el área se encuentran asociadas a estos farallones (peñas), de tal manera que el muro de piedra y barro que las cierra completamente se apoya sobre las paredes rocosas. En términos morfológicos, suelen tender a la forma troncocónica, con una planta subcircular con un diámetro promedio de un metro, y una altura también promedio de 1,20 me- tros $^{2}$. Todas las estructuras que hemos estudiado presentan una abertura cuadrangular, que define el frente, de unos 0,40 metros de lado. De todas maneras, existe una notable variabilidad asociada con las decisiones y agencias de los distintos constructores y vinculada también con la interacción necesaria entre una estructura y otra para formar los agrupamientos ${ }^{3}$.

2 A lo largo de todo el artículo, las medidas a las que se haga referencia corresponden a dimensiones exteriores máximas, salvo aclaración explícita en contrario.

3 En relación con la significación que tienen estas estructuras para las poblaciones actuales en el área, hemos decidido en conjunto con estas comunidades que en esta primera etapa de la investigación no se realizarían excavaciones arqueológicas ni en las estructuras ni en los agrupamientos. Aunque eventuales excavaciones futuras podrían ser productivas, esto no ha sido una limitante para aproximarse a los sentidos de estas construcciones a partir del análisis de sus características arquitectónicas. Por otra parte, cabe aclarar que no hemos observado en ninguna de las 101 estructura ni restos humanos ni objetos en superficie, estando todas completamente vacías en su interior. 

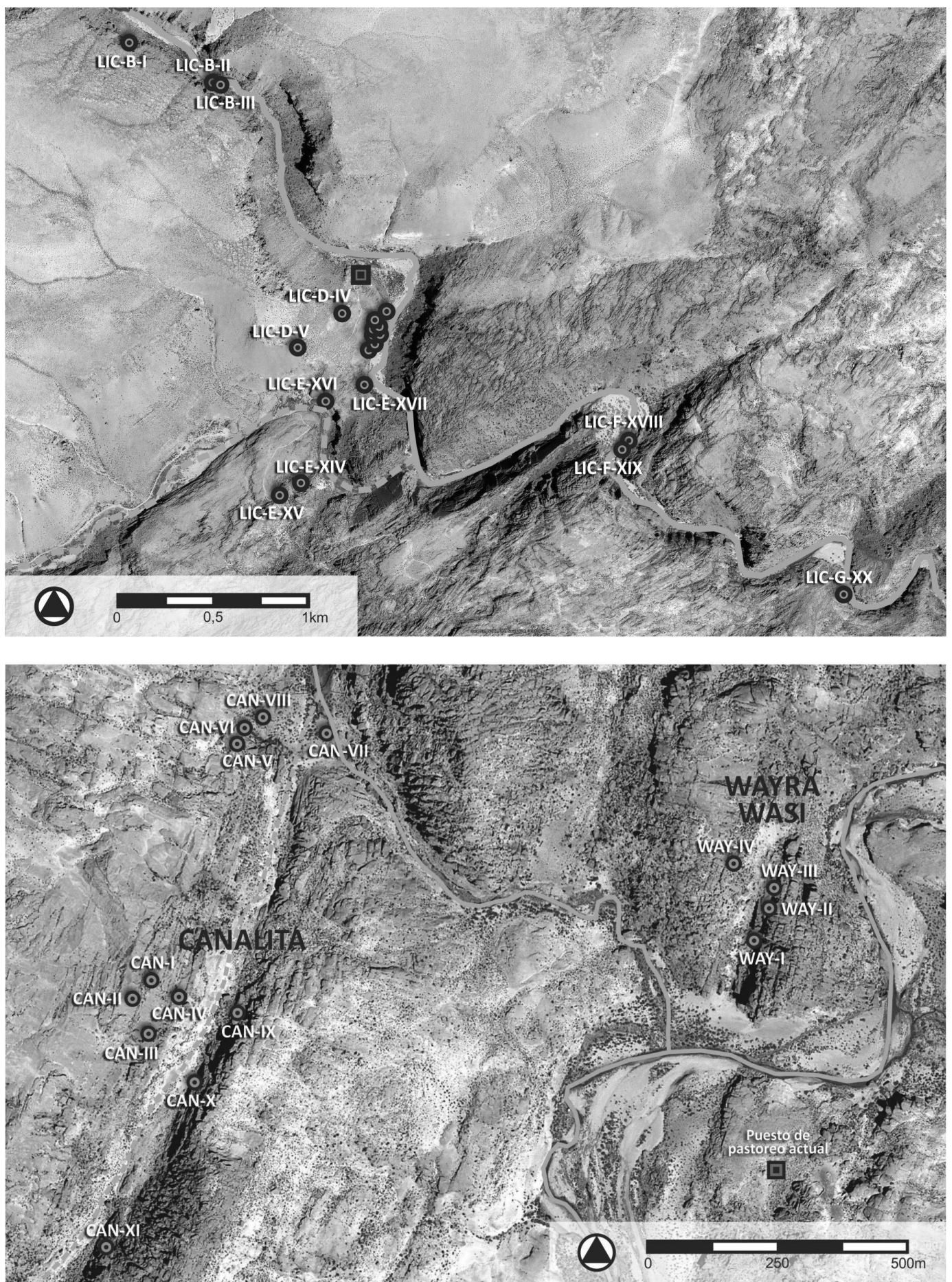

Figura 2. Agrupaciones chullparias en los sitios de Licante, Canalita y Wayra Wasi (Sección Agua Delgada, Coranzulí). 


\section{* LA CONDición AgRupada}

Para tener un primer acercamiento a la significación que tienen los agrupamientos para la definición de las estructuras chullparias en el área de estudio, debemos considerar que de las 101 estructuras que forman la muestra relevada, solo 15 (15\%) se presentan sin vínculos directos con otras. Las 86 restantes se encuentran formando 20 agrupamientos de entre 2 y 10 estructuras. Si discriminamos esta información por sitio, la muestra de Licante (59\% del total) está formada por 60 estructuras, de las cuales ocho aparecen solas (13\%) y las 52 restantes $(87$ $\%)$ se organizan en 12 grupos de entre 2 y 10 estructuras. La muestra de Canalita ( $28 \%$ del total) reúne 28 estructuras, de las cuales cinco se presentan solas (18\%) y las 23 restantes (82\%) se organizan en seis grupos de entre 2 y 7 estructuras. Por su parte, la muestra de Wayra Wasi ( $13 \%$ del total) consta de 13 estructuras, de las cuales dos aparecen solas $(15 \%)$ y 11 se agrupan $(85 \%)$ en dos grupos de 2 y 9 estructuras (Figura 2 ).

La definición de una serie de estructuras como agrupamiento surge de la combinación de los siguientes criterios: la existencia de un vínculo material explícito entre éstas, la cercanía estrecha entre estas construcciones y/o la orientación de sus aberturas. Al hablar de un vínculo material nos referimos a que dos o más estructuras compartan muros entre sí, mientras que en el segundo criterio consideramos que, ante la ausencia de aquel vínculo explícito, la separación entre estructuras no supera los tres metros. Finalmente, el tercer criterio, complementario del resto, está definido a partir de las aberturas, tal que se constituye como un agrupamiento cuando sus orientaciones generan áreas compartidas entre estructuras. En todo caso, cabe observar que si nos concentramos en las quince estructuras aisladas, cuatro están a menos de 10 metros de otras y otras ocho a menos de 50 metros, mientras que solo tres presentan una distancia superior. Lo que nos interesa marcar con esto es que, aunque no formen parte de agrupamientos, las estructuras chullparias en el área establecen algún tipo de relación entre sí, con una alta densidad de construcciones.

En relación con las características de la disposición espacial de las estructuras agrupadas, hemos definido tres tipos: Alineado, Celular y Superpuesto (Figura 3). La diferenciación de estos tres tipos es importante porque nos permite reconocer la existencia de distintas formas de uso del espacio y de diferentes relaciones entre las estructuras. Ciertamente el tipo Alineado se configura como el más habitual entre la muestra relevada, concentrando el 70\% de los grupos, mientras que el Celular alcanza el $20 \%$ y el Superpuesto el 10\%. Si bien, y como veremos más adelante, estos tipos pueden aparecer combinados entre sí, para discriminarlos hemos considerado el predominante.

El tipo de agrupamiento alineado es, por definición, un planteo lineal en el que se prioriza un eje longitudinal en el desarrollo en planta, más que la superposición de dos ejes, longitudinal y transversal, propia del agrupamiento celular. Esto implica que las estructuras se disponen una al lado de la otra, conformando un frente que, sin embargo, puede ser discontinuo. La relación entre las estructuras en esta configuración puede implicar, o no, que compartan muros entre ellas. Por otro lado, la totalidad de las estructuras en este tipo de agrupamientos se presentan apoyadas sobre la pared de una peña. Del total de 20 grupos registrados, 14 presentan este tipo de disposición espacial. En términos de cantidad de estructuras, 46 de las 101 (46\%), están involucradas en una configuración alineada. Si analizamos esta misma información

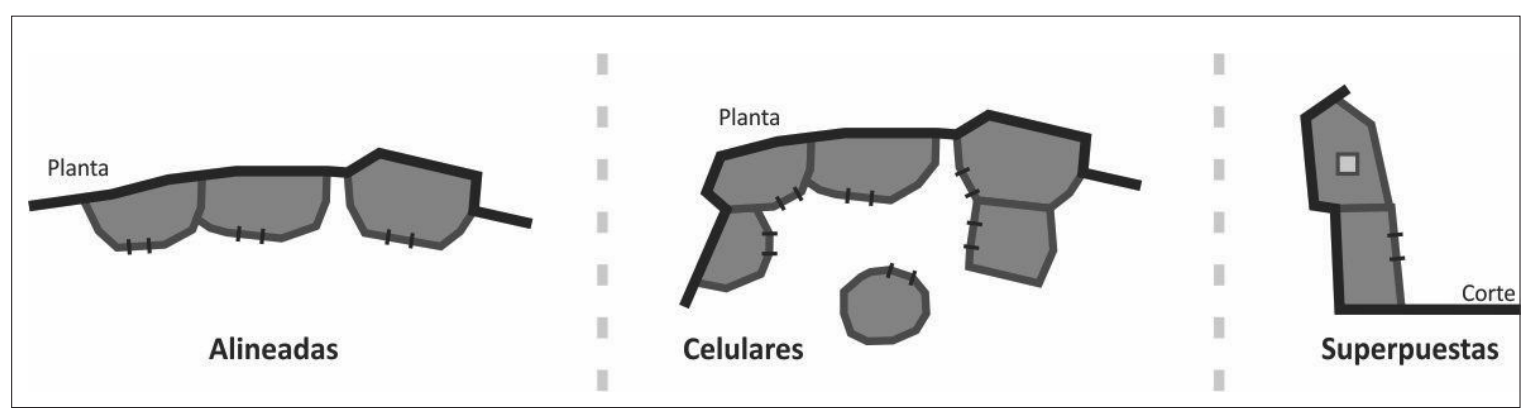

Figura 3. Tipos de agrupamientos chullparios propuestos para el área de estudio. 
discriminada por sitio, observaremos que en Licante, el $75 \%$ de los grupos ( $50 \%$ de las estructuras) corresponde a este tipo, mientras que en Canalita asciende al 67\% de los grupos (50\% de las estructuras) y en Wayra Wasi este tipo de agrupamiento alcanza el 50\% de los grupos (15\% de las estructuras).

Un caso representativo es un agrupamiento en el sitio Licante (LIC-D-X) que se compone de seis estructuras alineadas, tres completas y el resto derrumbadas. Están apoyadas sobre la pared de la peña, en la parte media de la ladera. Cinco de estas chullpas están dispuestas de tal manera que comparten sus muros laterales, mientras que la restante está separada. Otro caso para considerar es un agrupamiento en Canalita (CAN-I) compuesto por siete estructuras alineadas que comparten al menos uno de sus muros, en una disposición encadenada. La pared rocosa, sobre la que se apoyan las siete, tiene un trazado curvo, resultando en una disposición del agrupamiento en arco (Figura 4). Es interesante considerar que, aunque las estructuras terminan conformando un frente común relativamente continuo, eso no implica una total homo- geneidad en las técnicas constructivas. Por el contario, se distinguen notables diferencias entre éstas, que podrían vincularse con diferentes constructores en distintos momentos (Rivet 2013b).

Nos referimos a un tipo de agrupamiento celular cuando una o más estructuras se disponen por delante de otras, sin estar apoyadas sobre la peña, aunque pudiendo compartir muros con las estructuras anteriores. Frente a la linealidad característica del tipo de agrupamiento anterior, aquí encontraremos conformaciones en las que se ponen en juego dos dimensiones espaciales en planta, y en ciertos casos una tercera en vertical. En lugar de un único espacio delante de las estructuras alineadas, acá se registra una suma de distintos espacios de vinculación. En estas configuraciones celulares, entonces, se enfatizan las relaciones, de alguna manera "diálogos", interestructuras.

Del total de 20 grupos, cuatro presentan este tipo de agrupamiento, lo que representa el $20 \%$ de la muestra. Cabe notar que en tres de estos casos, dentro de la organización celular general, existen relaciones alineadas

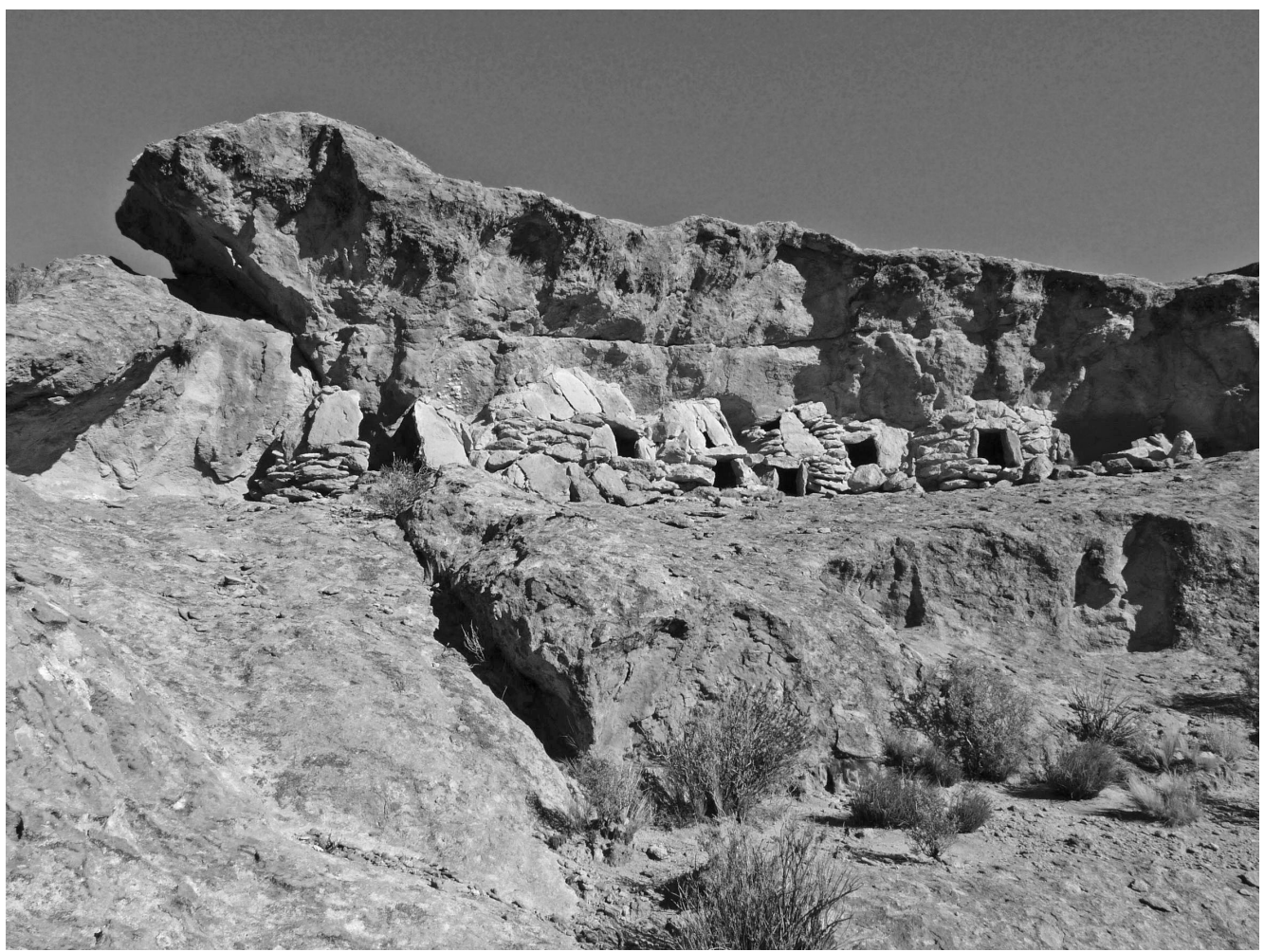

Figura 4. Ejemplo de agrupamiento chullpario, tipo alineado, en Canalita (CAN-I). 
e incluso superpuestas, entre algunas de las estructuras. Por otra parte, todos se desarrollan al interior de aleros rocosos, lo que le da ciertas características particulares. Uno de los grupos en Wayra Wasi (WAY-IV) es representativo: se compone de nueve estructuras ubicadas dentro de un alero, que se abre al este, de base muy irregular, con desprendimientos de bloques de la ignimbrita en su interior, lo que permite la utilización de distintos niveles para las construcciones (Figura 5). De estas nueve chullpas, seis están apoyadas sobre las paredes rocosas, mientras que dos están adosadas a otras preexistentes y la restante es independiente. De esta disposición surgen tres espacios de vinculación, en el sentido que lo referimos más arriba.

El tipo superpuesto corresponde a aquellos agrupamientos cuyas estructuras se emplazan una encima de la otra. Es decir, el desarrollo principal no se da horizontal sino verticalmente. Esta disposición surge principalmente del aprovechamiento de irregularidades topográficas, que facilitan el asentamiento en distintos niveles. Si bien solo dos casos (10\%) se agrupan de esta manera, uno en Licante (LIC-E-XV) y el otro en Canalita (CAN-VII), lo cierto es que aparecen desarrollos verticales en el marco de agrupamientos que son fundamentalmente celulares, e incluso en alineados. En este último punto nos referimos a aquellas estructuras que, si bien plantean un uso vertical del espacio, no están vinculadas constructivamente con otras. Este factor es excluyente para definir los grupos superpuestos como tales. El agrupamiento consignado en Canalita (CAN-VII) se compone de seis estructuras ubicadas en la base del farallón, aprovechando el interior y exterior de una oquedad en la peña (Figura 6). Tres de estas estructuras se encuentran dentro de ésta, dispuestas de forma escalonada en distintos niveles, superpuestas en sentido estricto. Es decir, el piso de una estructura es coincidente con el nivel del techo de la otra, superponiéndose incluso parcialmente.

\section{* Secuencias constructivas}

El estudio de la conformación de estos agrupamientos nos brinda la posibilidad de aproximarnos a los procesos de construcción en el tiempo, proponiendo una serie de secuencias constructivas, sobre la base del análisis de distintas líneas diferentes de evidencias. Es así como tomaremos los fechados radiocarbónicos ${ }^{4}$ realizados sobre la

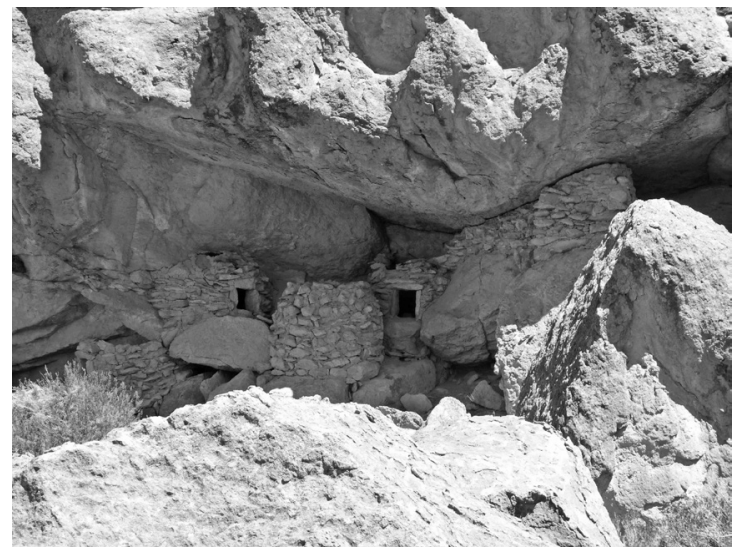

Figura 5. Ejemplo de agrupamiento chullpario, tipo celular, en Wayra Wasi (WAY-IV).

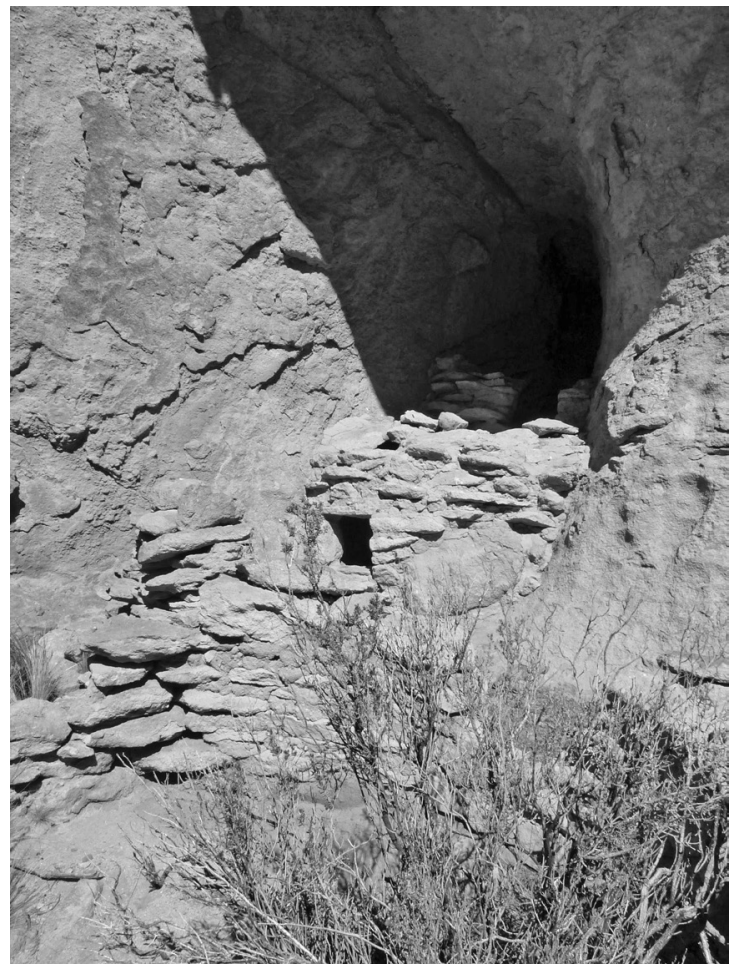

Figura 6. Ejemplo de agrupamiento chullpario, tipo superpuesto, en Canalita (CAN-VII).

4 La totalidad de los fechados fueron realizados en el NSF-Arizona AMS Laboratory, University of Arizona. Le agradecemos al Dr. Catriel Grecco, quien realizó las calibraciones de los fechados, utilizando el programa OxCal v4.1.7 (Bronk Ramsey 2009). 


\begin{tabular}{|c|c|c|c|c|c|c|c|}
\hline & Estructura & $\begin{array}{l}\text { Código Labo- } \\
\text { ratorio }\end{array}$ & $\mathrm{d}_{13} \mathrm{C}$ & $\begin{array}{l}\text { Años } \mathrm{C}_{14} \\
\mathrm{AP}\end{array}$ & $\begin{array}{c}\text { Rango de años calibra- } \\
\text { dos DC } \\
68,2 \% \text { de probabilidad }\end{array}$ & $\begin{array}{c}\text { Rango de años calibra- } \\
\text { dos DC } \\
95,4 \% \text { de probabilidad }\end{array}$ & Material \\
\hline \multirow{4}{*}{$\frac{\overrightarrow{0}}{\dot{u}}$} & LIC-B-I-ol & $\mathrm{AA} 98423$ & -16.6 & $619 \pm 36$ & $\begin{array}{l}1320(36.0 \%) 1350 \\
1386(32.2 \%) 1411\end{array}$ & $\begin{array}{l}1304(49.8 \%) 1363 \\
1377(45.6 \%) 1428\end{array}$ & Gramínea \\
\hline & LIC-B-I-O2 & AA93749 & -20.6 & $605 \pm 33$ & $\begin{array}{l}1325(21.2 \%) 1342 \\
1390(47.0 \%) 1415\end{array}$ & $\begin{array}{l}1315(35.3 \%) 1358 \\
1381(60.1 \%) 1435\end{array}$ & Gramínea \\
\hline & LIC-B-I-1O & AA9 8422 & -11.9 & $650 \pm 36$ & $\begin{array}{l}1314(49.9 \%) 1358 \\
1380(18.3 \%) 1398\end{array}$ & $1298(95.4 \%) 1405$ & Gramínea \\
\hline & LIC-B-I-10 & AA93748 & -23.0 & $119 \pm 33$ & $\begin{array}{l}1705(9.0 \%) 1722 \\
1810(17.5 \%) 1838 \\
1846(10.5 \%) 1867 \\
1878(31.2 \%) 1928\end{array}$ & $\begin{array}{l}1690(16.5 \%) 1728 \\
1805(78.9 \%) 1952\end{array}$ & Gramínea \\
\hline \multirow{3}{*}{ 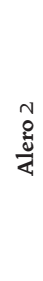 } & $\begin{array}{l}\text { Muro } \\
\text { Perimetral LIC- } \\
\text { D-XI }\end{array}$ & $\mathrm{AA} 98424$ & -23.1 & $654 \pm 36$ & $\begin{array}{c}1313(50.8 \%) 13581380 \\
(17.4 \%) 1397\end{array}$ & $1296(95.4 \%) 1405$ & Gramínea \\
\hline & LIC-D-XI-O3 & AA93747 & -11.7 & $617 \pm 33$ & $\begin{array}{c}1322(34.1 \%) 13481387 \\
(34.1 \%) 1410\end{array}$ & $\begin{array}{c}1310(47.3 \%) 13601378 \\
(48.1 \%) 1427\end{array}$ & Gramínea \\
\hline & LIC-D-XI-O5 & AA98425 & -25.1 & $569 \pm 41$ & $1397(68.2 \%) 1437$ & $\begin{array}{c}1320(11.5 \%) 13501386 \\
(83.9 \%) 1451\end{array}$ & Gramínea \\
\hline
\end{tabular}

Tabla 1: Fechados calibrados con curva del Hemisferio Sur (ShCalo4)

paja recuperada del mortero de barro de seis estructuras (Tabla 1), las características del ensamble entre estructuras, las diferencias macroscópicas de la argamasa y el tratamiento de las piedras utilizadas en cada construcción.

En relación con el ensamble entre las estructuras, es necesario que observemos ciertos rasgos generales que son los que nos permitirán reconocer preexistencias y adiciones. Como hemos adelantado, el cierre completo de la caja muraria es una condición excluyente en la construcción de las estructuras en el área. Esto implica que aquellas que fueron construidas en primer término presentan un muro continuo en todo su perímetro. Al sumarse una nueva estructura, adosada a una preexistente, pareciera haber una regla general respecto al modo en que se realiza esta operación. Los muros de la estructura que se adosa se realizan exclusivamente hasta el encuentro con la construcción anterior. Es decir, el cerramiento de esta nueva estructura se completa con el preexistente, por lo que no se observa una duplicación de los muros. Aquella cara que era exterior en la estructura original, deviene en interior de la que se adosa. Esto implica, a su vez, una transformación sobre la expresión y terminación de este muro. En muchos casos se ha cargado con barro adicional para lograr juntas al ras, donde antes, cuando era exterior, estaban tomadas (Rivet 2014a). Este no es un detalle menor puesto que conlleva un cambio en la condición de este muro, además de ser una evidencia de la secuencia constructiva. Por otra parte, debemos observar que la construcción que se suma a una preexistente se apoya literalmente en ésta, de tal manera que en cierto modo se vuelve estructuralmente dependiente. En otros términos, un eventual colapso de la primera, implicaría también el derrumbe de la segunda.

En todos los casos registrados los encuentros entre los muros se realizaron sin traba. Es decir, no se retiraron piedras para mejorar el anclaje, aunque sí se observa la 
adición de abundante mortero para esconder el encuentro. Esto se evidencia particularmente cuando, producto de asentamientos diferenciales de las estructuras, estos encuentros presentan fracturas en todo su largo. Como ha planteado Taboada (2005), para el análisis de las correlaciones en las arquitecturas domésticas, esta ausencia de traba es un indicador de un ordenamiento diacrónico de las construcciones.

A partir de estos elementos, nos interesa aproximarnos a las posibles secuencias constructivas dentro de tres agrupamientos. Dadas sus características, se trata de casos representativos y significativos dentro de la muestra de estudio que nos permitirán aproximarnos a situaciones con diferentes niveles de complejidad. Estos tres agrupamientos que consideraremos se ubican dentro de aleros rocosos, completamente cubiertos, y dos presentan arte rupestre prehispánico, colonial y republicano en asociación con las estructuras chullparias (Rivet 2013b). Este recorrido nos permitirá proponer posibles secuencias a partir del análisis de las diferentes etapas dentro de la construcción en el tiempo de un agrupamiento.

Caso 1. Este agrupamiento se construyó dentro de un alero (Alero 1), ubicado en la base de un farallón, con una boca orientada al norte y una planta prácticamente rectangular. En su pared posterior presenta una saliente, a tres metros de altura, que forma una especie de escalón, con una pendiente combinada hacia el este y el norte. En el interior se han construido diez estructuras chullparias, dispuestas en dos niveles, el primero sobre el piso del abrigo, y el segundo con una única estructura, en la saliente rocosa (Figura 7). Todo este conjunto de estructuras es contenido por un muro frontal, que en parte se encuentra derrumbado.

Las nueve estructuras del nivel inferior están dispuestas de acuerdo a una serie de criterios. En primer lugar están "encadenadas" compartiendo muros y a su vez, siete de éstas se ubicaron apoyadas sobre todo el perímetro interno del alero, ambos laterales y la pared posterior, mientras que las dos restantes avanzan sobre el centro, adosadas sobre las anteriores. Dos de las chullpas (05 y 07) ubicadas en el perímetro, se encuentran en un nivel intermedio sobre bases tipo pedestal. A pesar de la densidad de construcciones, la disposición permite llegar a todas las estructuras. La única excepción es la estructura del segundo nivel (10), puesto que es necesario subir al resto de las chullpas para acceder a ella. Las paredes de este alero fueron usadas como soporte de arte rupes-

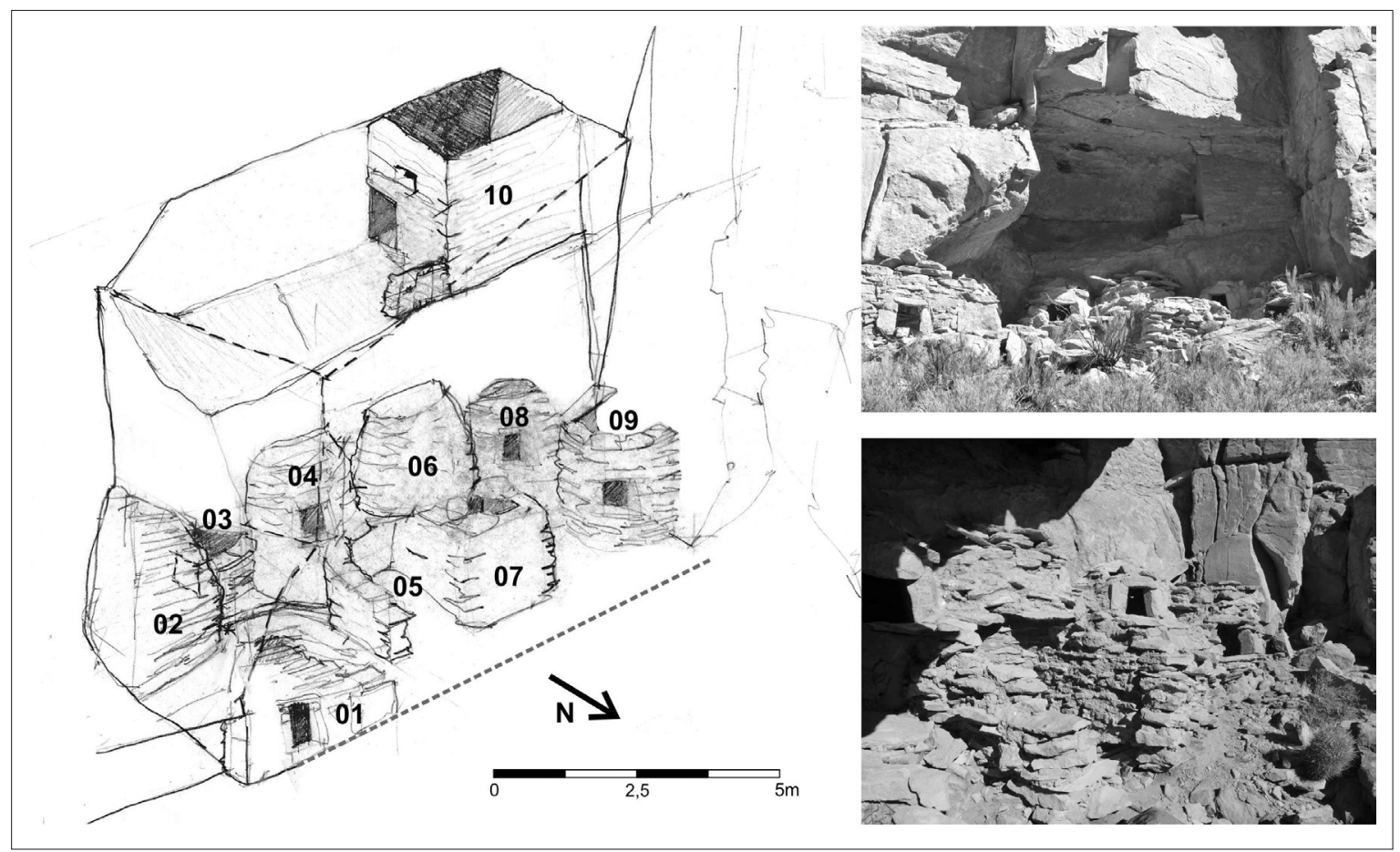

Figura 7. Croquis y fotos del Alero I (Licante). 
tre, tanto prehispánico como colonial y republicano. En cuanto a los primeros, se trata de pinturas ubicadas en los sectores bajos de la pared de la peña que fueron envueltos por las estructuras chullparias (Rivet 2013b). En cuanto a las representaciones ejecutadas en momentos coloniales, se distinguen pinturas en la pared del alero y grabados en el barro de la estructura en el nivel superior, la mayoría de éstas de índole cristiana (Rivet 2013a). Por otro lado, hemos identificado ciertas grafías que podrían corresponder a marcas de ganado, ya en tiempos republicanos. Éstas fueron realizadas tanto en las paredes rocosas como en las jambas de algunas de las estructuras chullparias.

El Alero 1 nos presenta para este análisis uno de los escenarios más complejos, producto de la cantidad de estructuras en distintos niveles y las numerosas superposiciones. Para este agrupamiento contamos con cuatro fechados: uno para la estructura LIC-B-I-ol, otro para LIC-B-I-O2, y los dos restantes para LIC-B-I-1o (ver Tabla 1). Estos fechados, en primer lugar, posicionan el comienzo de la construcción de este agrupamiento en el siglo XIV y, a su vez, evidencian intervenciones posteriores correspondientes al siglo XVIII/XIX. Dentro del análisis constructivo proponemos que las estructuras 04 y 09, por sus características, podrían haber sido las primeras en realizarse dentro del Alero (Ver esquema del proceso en la Figura 8). Las dos tienen una caja muraria continua que cierra todo el perímetro, sin adosarse sobre ninguna otra. Esto no implica que hayan sido contemporáneas, ni brinda información sobre el orden en que podrían haberse construido. A partir de esto, podemos observar una serie de condiciones de superposición que permiten reconstruir parcialmente la secuencia total.

En primer lugar, la estructura o1, sobre la que tenemos un fechado y que a la vez conforma parcialmente el muro frontal del alero, se habría elevado con anterioridad a la O2, que también hemos datado. A su vez, esta estructura O2 se adosa sobre 03, puesto que se distingue cómo el muro lateral izquierdo de esta última es continuo hasta la pared rocosa (Figura 9). Finalmente, la estructura $\mathrm{O}_{3}$ se adosa sobre la 04 , sobre el muro lateral izquierdo de ésta (Figura 10). Por el otro lado, el desarrollo de la estructura o6 se apoya sobre el de la 04, marcando su realización posterior. A su vez, ambas tienen una base pedestal hasta la misma altura, reconociéndose cómo, en la base de 04 , se utilizó un aparejo encastrado mientras que la de 06 es en hiladas. Además de esta diferencia se reconoce cómo la primera es continua mientras solo se adosa. En el encuentro de los desarrollos de estas dos estructuras ( 04 y o6) se observa un posible agregado posterior de piedra con barro que podría haber actuado como escalón para alcanzar el nivel de la estructura 10.

La estructura 08 , a su vez, se adosa sobre la 09, a partir de la discontinuidad del muro de la primera y la ausencia de traba en el encuentro. No es posible observar con claridad la preexistencia entre las estructuras o 8 y 06, aunque sí cabe señalar que la primera presenta una base pedestal de la misma altura que 04 y 06 . De hecho, el desarrollo de las tres comienza en el mismo nivel y se

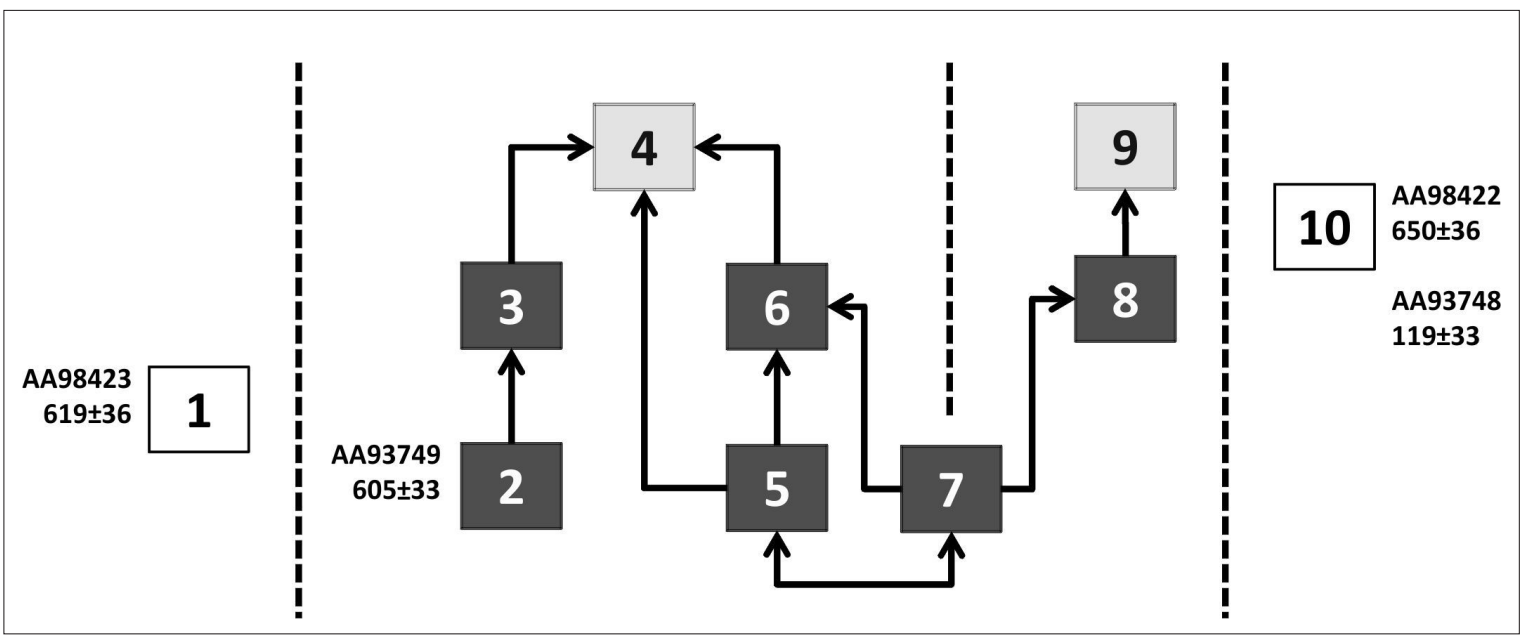

Figura 8. Esquema de las secuencias constructivas del Alero 1. 


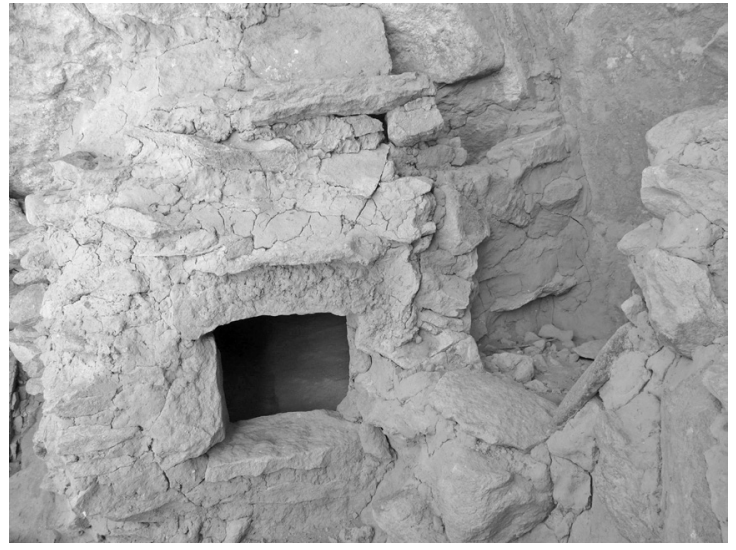

Figura 9. Apoyo de la estructura 02 , a la izquierda, sobre el muro de la 03 , en el Alero 1.

observa una suerte de entrepiso plano que las vincula. Mientras que todas las estructuras mencionadas hasta el momento se encuentran apoyadas, la 05 y la 07 son adosadas y entonces no tienen un vínculo directo con la pared del Alero. En particular la 07 se adosa a las bases de o6 y o8, mientras que la 05 lo hace con 04 y o6, lo que marca con claridad que son posteriores. El muro frontal de 05 y 07 pareciera ser continuo y no presenta traba en el encuentro del muro que divide a ambas. Esto abre dos posibilidades: que se hayan construido simultáneamente o que el muro frontal sea una construcción posterior. Finalmente la estructura 10 no es dependiente de ninguna de las otras, de hecho está en un nivel distinto, y configura un problema en sí mismo, puesto que es una de las cuatro estructuras con doble abertura de la muestra total, contando además con un fechado que posicionaría su construcción en momentos coloniales tardíos o ya republicanos (Rivet 2013a) 5

En síntesis, partiendo de la estructura 04 se observan dos sucesiones de nuevas construcciones. Por un lado, hacia su izquierda, el encadenado 03 y 02 , y por el otro, la 06. A su vez, a partir de la estructura og se sumó la 08, siendo que la 07 se adosó a la 08 y 06 y la 05 a la 06 y 04 . Esto implica que el fechado sobre la estructura ol no corresponde a las primeras operaciones dentro del Alero, aunque tampoco tuvo porqué haber sido la última. El bloque 03-02 podría perfectamente haberse construido antes del 06-05-07, ya que son independientes entre sí. A partir de esta reconstrucción parcial de la secuencia constructiva del Alero, podemos esbozar al menos dos

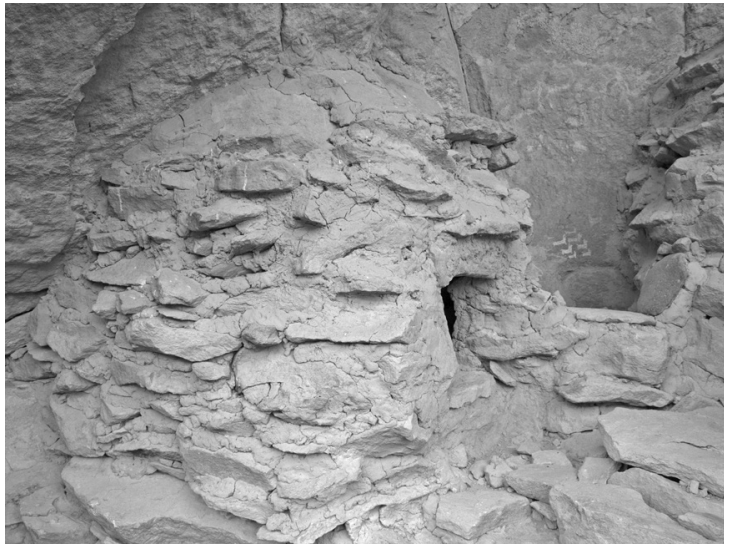

Figura 10. Apoyo de la estructura 03, sobre la O4, en el Alero 1.

reflexiones. Por un lado, más allá de cuál o cuáles hayan sido las estructuras iniciales, es claro que las primeras construcciones se realizaron en relación directa con las paredes del alero. Recién luego se habría ocupado el espacio central, separado de las paredes rocosas, al haberse cubierto la totalidad del perímetro ${ }^{6}$. Esto es congruente con la preferencia general en el área (por encima del 85\%) de construir las estructuras apoyadas sobre las paredes de las peñas.

La segunda reflexión a plantear es que la imposibilidad de reconocer vínculos y superposiciones entre todas las estructuras, en lo que sería un único "encadenado conti-

5 Vale aclarar que contamos con dos fechados para esta estructura (ver Cuadro 1), uno tardocolonial y otro correspondiente aproximadamente al siglo XIV. Ambos fueron realizados a partir de muestras de gramíneas tomadas del mortero de barro. Dadas las características morfológicas de esta estructura, que se diferencia del resto remitiendo a ciertos rasgos de la arquitectura eclesiástica, nos inclinamos por posicionar su construcción en momentos coloniales tardíos. Por otro lado pensamos que el desfasaje en las fechas puede corresponder a un evento de reutilización del barro de otras estructuras prehispánicas. Hemos desarrollado el problema de esta estructura en Rivet 2013 a y b.

6 La falta de toda la secuencia constructiva, que podría completarse con nuevos fechados en el futuro, nos obliga a una salvedad respecto a esta sentencia. Como se aclaró, en el sentido inverso, las estructuras 01, 02 y 03 podrían eventualmente ser posteriores a 05 y 07, puesto que no hay ningún encuentro que clarifique las precedencias. En este caso, la pared este del alero, se habría completado cuando ya existían estructuras en el espacio central. 


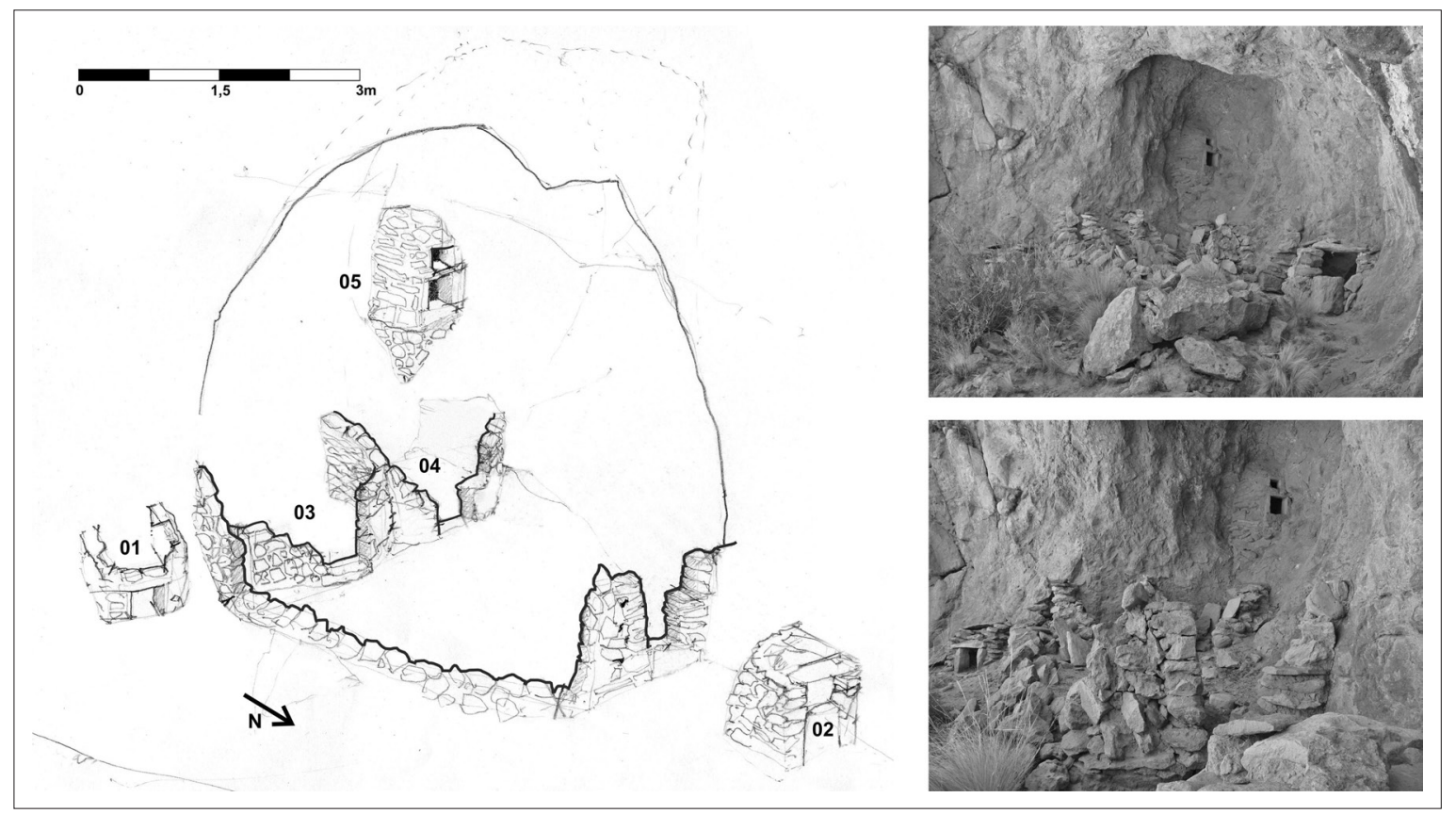

Figura 11. Croquis y fotos del Alero 2 (Licante).

nuo", es un dato en sí mismo y nos lleva a proponer lo que denominaremos "líneas de crecimiento". Con esto nos referimos a que la construcción de nuevas estructuras se dio en distintos sectores con una relativa independencia. $\mathrm{La} / \mathrm{s}$ persona/s habrían tenido márgenes de elección respecto a cuál de las estructuras adosarían la nueva construcción o directamente si la elevaban independiente del resto. Es así como se reconoce una línea de crecimiento 04-03-02, otra 04-06-05-07 y otra 09-08-07, sin que puedan reconocerse por el momento los vínculos cruzados entre las tres. Ciertamente esto abre una pregunta importante, que intentaremos profundizar luego, respecto a las razones que llevaban a preferir un vínculo, dentro de una "línea de crecimiento", por encima de otro. Por el momento, es importante que consideremos que la construcción del agrupamiento no se habría dado en un único evento, sino que estaríamos ante diferentes intervenciones, más o menos independientes, dentro de una única lógica global de agrupamiento.

Caso 2. Este agrupamiento (Alero 2) está ubicado a 1,5 km del anterior, en la parte media de un farallón de ignimbritas, a unos 20 metros sobre el nivel del río. El alero tiene una boca de forma pseudoparabólica, orientada hacia el este, con una planta irregular que tiende a lo triangular.

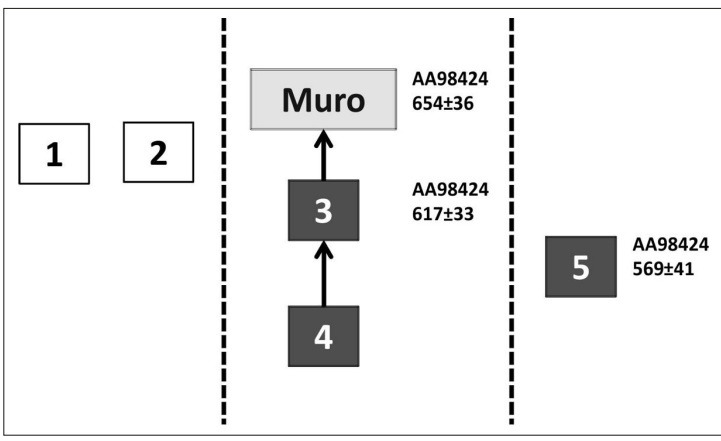

Figura 12. Esquema de la secuencia constructiva del Alero 2.

El espacio interior fue delimitado como tal a partir de la construcción de un muro frontal de piedras y argamasa de barro, que cierra la boca del alero. Este muro de cerramiento es continuo aunque se presenta derrumbado en su porción media. Se reconoce con claridad una abertura de ingreso orientada hacia el norte, con un pequeño escalón en el umbral (Figura 11). Las cinco estructuras chullparias de este alero se distribuyen dos hacia el exterior, en las esquinas norte y sur, y tres en el interior. Las dos primeras ( $\mathrm{O} 1$ y O2) son de menores dimensiones que el resto y se emplazan apoyadas sobre la pared rocosa, con sus aberturas orientadas al norte. Dentro de las tres interiores, dos (03 y 04) están dispuestas en el piso del alero, 


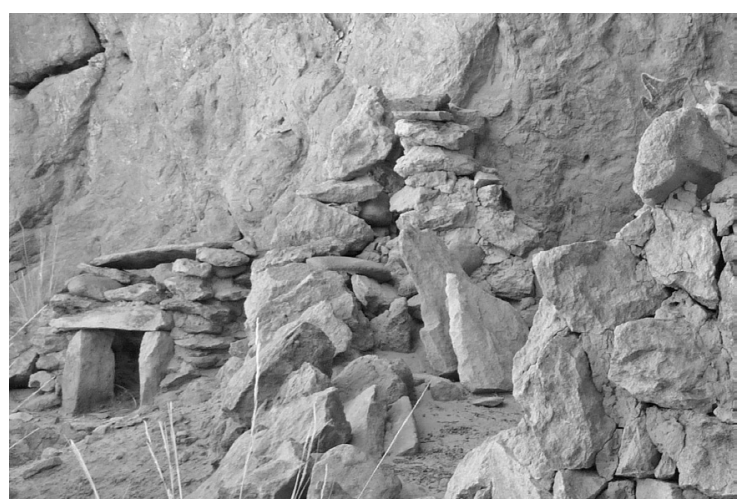

Figura 13. Encuentro sin traba entre la estructura 03 y el muro perimetral, en el Alero 2. de momentos prehispánicos y coloniales. Se trata de siete conjuntos que se ubican por dentro y por fuera de las estructuras chullparias. En particular sobresale en la pared norte, un panel compuesto por 56 personajes alineados, con diferentes vestimentas y tocados. Cinco de los conjuntos están ubicados en el interior de la estructura 03.

Para este caso contamos con tres fechados: uno sobre el muro perimetral, y dos sobre las estructuras 03 y 05 (ver Tabla 1), que muestran intervenciones en lapsos temporales relativamente cortos. Si lo consideramos en relación con los datos constructivos, podemos observar que la elevación del muro perimetral sería anterior a las

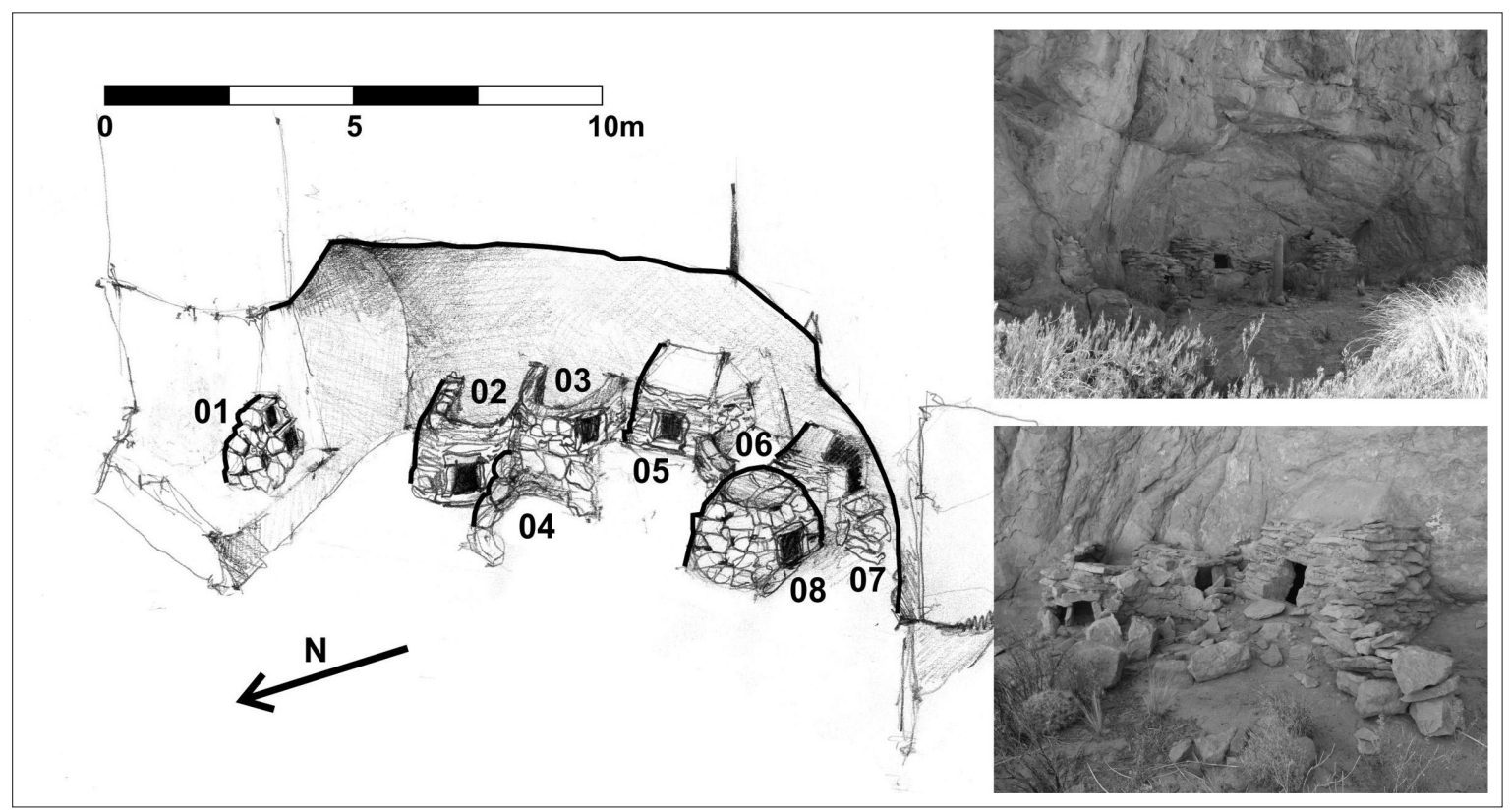

Figura 14. Croquis y fotos del Alero 3 (Licante).

apoyadas sobre la pared sur, y compartiendo uno de sus muros laterales, con vanos abiertos al norte. A su vez, en la estructura 03 el cerramiento lateral izquierdo es coincidente con el muro frontal del alero. Estas dos estructuras son de mayores dimensiones que el promedio de las relevadas entre los tres sitios. La quinta estructura (05) se ubica en una pequeña saliente, elevada, en la pared posterior, sobre una base de nivelación. Al igual que la estructura 10 del alero 1, ésta también posee dos aberturas, una más grande, y la otra más pequeña encima. Este alero presenta una notable cantidad y variedad de arte rupestre en todas sus paredes, incluyendo representaciones estructuras ubicadas en el interior. La estructura $\mathrm{O}_{3}$, además de estar apoyada sobre la pared rocosa, se adosa a este muro, que se constituye como su cerramiento lateral izquierdo. En el encuentro entre ambos se observa una fractura importante que evidencia la falta de traba. La estructura 04 , a su vez, se adosa a la 03 , siendo entonces posterior. Finalmente, el fechado sobre la estructura ubicada en el nivel superior, que no tiene vínculo constructivo con las anteriores, evidencia que ésta habría sido posterior tanto al muro como a 03. Las dos estructuras restantes ( $\mathrm{O} 1$ y o2) ubicadas a ambos lados en el exterior del alero, nos presentan una situación particular, puesto 
que no se correlacionan constructivamente con ninguna de las estructuras del interior ni con el muro perimetral, a la vez que tampoco tenemos al momento dataciones absolutas (Figura 12).

La proximidad temporal entre los tres fechados obtenidos nos permite posicionar la construcción de este agrupamiento en torno al siglo XIV (y potencialmente principios del XV), pero no nos brinda precisión respecto a la sucesión de eventos. Es aquí donde los datos constructivos (cruzados con estos fechados) se vuelven importantes. El muro perimetral se habría elevado antes que las dos estructuras 03 y 04 , puesto que la primera apoya sin traba sobre dicho muro, y lo propio ocurre con la 04 en relación con la 03 (Figura 13). Al respecto cabe observar que la decisión de cerrar el alero no habría sido accesoria, ni posterior, sino que es constitutiva en la definición inicial del agrupamiento. A su vez, gran parte de los conjuntos de arte rupestre ya habrían sido ejecutados en las paredes del alero, y entonces fueron envueltos por este muro. Se vuelve estimulante pensar en un espacio que ya era significativo antes de la elevación de las chullpas, y con estas nuevas materialidades esos sentidos se habrían sostenido y reforzado.

Caso 3. Este agrupamiento (Alero 3) se ubica a 1,5 km, aguas abajo, del anterior y está emplazado en la base de un farallón. La boca del alero se abre hacia el noroeste y su piso se encuentra alrededor de tres metros por encima del nivel del río, con una ligera pendiente hacia el exterior, de manera tal que la parte posterior es algo más elevada que la frontal (Figura 14). En su interior se construyeron ocho estructuras chullparias que presentan características ligeramente diferentes entre sí, evidenciándose, por las superposiciones de los muros, distintos momentos de construcción. De estas ocho, seis están ubicadas en forma alineada, apoyadas sobre la pared posterior, mientras que otras dos se emplazan por delante, y se encuentran adosadas a éstas, ocupando la porción central del alero. No hemos registrado ningún cerramiento frontal, como se observa en los dos casos anteriores. El arte rupestre también está ausente en este espacio.

La estructura or presenta doble abertura y se ubica en el extremo izquierdo de la boca del alero, sobre un escalón natural de ignimbrita, que si bien la eleva $0,70 \mathrm{~m}$ sobre el nivel de base, no es exactamente la misma situación que la de los aleros anteriores. En relación con el resto de las estructuras cabe observar que la 02 y la 03 se apoyan sobre la pared rocosa y a su vez comparten un muro lateral entre sí, mientras que la 04 se adosa a éstas, particularmente a la 03 . La estructura 05 fue construida, de alguna manera, en el centro de la pared posterior del alero, sobre la que se apoya, compartiendo además su muro lateral con la o6. Esta última, a su vez, comparte otro muro con la estructura 07, que también se encuentra apoyada. Finalmente, la estructura 08 se encuentra adosada a la 07. Como se observa, aunque existen distintos vínculos entre las construcciones, no todas están interrelacionadas entre sí.

Es posible reconocer tres estructuras que podrían haber sido las iniciales: 03, 05 y 07, todas apoyadas sobre la pared rocosa. A partir de estas tres se fueron desarrollando distintas líneas de crecimiento. La estructura $\mathrm{O} 2$, se encuentra apoyada sobre la 03, aunque en un nivel 0,30 metros más bajo. En el caso de la estructura 04 , construida también en un nivel ligeramente más bajo, el discernimiento es más complejo, puesto que es independiente, observándose un reducido espacio continuo que la separa de 02 y 03. De todas maneras, por su emplazamiento y el vínculo morfológico que se establece, es posible pensar que es posterior a estas dos. Por otro lado, la estructura o8 se encuentra adosada a la 07 , pudiendo reconocerse el ensamble no en el desarrollo sino en la base.

La estructura 06 se emplaza en el espacio entre 05 y 07 , utilizando como propios sus muros laterales, siendo entonces posterior a ambas. Finalmente, la estructura $\mathrm{OI}$, con doble abertura, nos presenta un escenario similar al

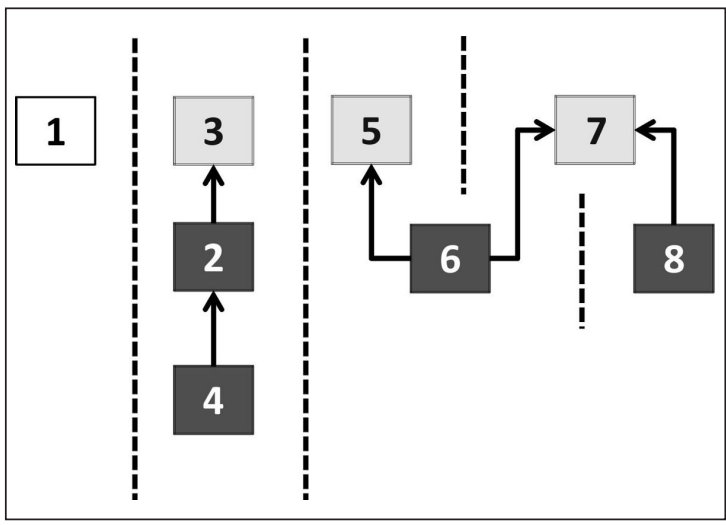

Figura 15. Esquema de las secuencias constructivas del Alero 3. 
de los dos aleros anteriores al no presentar vínculos con el resto de las construcciones del alero. En este sentido, no es posible ubicarla dentro de la secuencia. En síntesis, es posible proponer tres líneas de crecimiento: 03-0204, 07-08-06 y 05-06, siendo que estas dos últimas concatenaciones presentan un vínculo entre sí. Este alero nos permite enfatizar lo observado en el caso 1 , respecto a la existencia de múltiples secuencias en el proceso de construcción de un agrupamiento (Figura 15).

\section{Espacialidades chullparias}

Estos tres casos nos permiten reconocer cómo los agrupamientos se constituyen como tales a través de un proceso en el tiempo, en el que nuevas estructuras se van sumando en vinculación explícita con las existentes. Aunque este análisis se podría desarrollar con otros tipos de agrupamientos, aquellos ubicados en los aleros nos brindan un campo particularmente interesante dada la cantidad de estructuras involucradas y las relaciones complejas que se establecen entre éstas. La organización espacial en estos aleros, ha sido, por definición, una realidad dinámica como lo muestran las secuencias que hemos desarrollado. Por otra parte, las evidencias indicarían que la ubicación de las nuevas estructuras no era aleatoria sino que estaba asociada con el énfasis de ciertos vínculos espaciales y arquitectónicos en el marco de los agrupamientos. Ciertamente debemos considerar la existencia de una lógica que podría estar asociada con diferentes vínculos sociales entre los ancestros, a través de las chullpas, y entre éstos y las personas.

En este marco, al hablar de espacialidades chullparias nos estamos refiriendo a un conjunto de decisiones, intervenciones y prácticas asociadas que hacen a la concepción, percepción y vivencia del espacio, en el sentido de Soja (1971), que en este caso estarían íntimamente vinculadas con el culto a los ancestros. Podemos reconocer diferentes rasgos arquitectónicos, en la conformación de los aleros, que hacen a estas espacialidades. Entre éstos nos interesa analizar brevemente la conformación de ciertas áreas en la que se integran las distintas estructuras, en relación con las "líneas de crecimiento"; la definición de ciertas circulaciones que permiten un recorrido; el aprovechamiento de diferentes niveles, a través de escalones o salientes rocosas y la presencia de cerramientos frontales en algunos de los aleros. Estas prácticas arquitectónicas caracterizan el espacio interior de los aleros, habilitando usos diferenciales del espacio, produciendo lugares altamente significados y significantes. Como veremos luego, esto nos permitirá pensar a los agrupamientos asociados a un cierto "proyecto", sujeto a transformaciones, que se desarrolla en el tiempo, más que en una mera suma de decisiones e intervenciones completamente autónomas. Lo que nos proponemos en este punto es precisamente observar estos aspectos que hacen a la organización espacial en los agrupamientos en general, y en los aleros en particular.

\section{Áreas de interacción}

A lo largo del análisis de las secuencias, hemos observado cómo la ocupación del espacio no sigue un concatenamiento lineal, donde el orden constructivo sería O1-O2-03, etc. Por el contrario, pareciera responder a otras lógicas, vinculadas a lo que hemos definido como "líneas de crecimiento". Es decir, distintos encadenamientos, más o menos independientes entre sí, en el marco de un mismo agrupamiento, que presentan una sucesión de construcciones. A partir de estructuras iniciales apoyadas sobre las paredes rocosas, otras se vinculan a éstas ocupando distintos sectores. Esta lógica de organizar el espacio va generando en el tiempo lo que podríamos denominar "áreas de interacción". Es decir, una serie de espacios abiertos delimitados por una cierta cantidad de estructuras vinculadas morfológica y constructivamente entre sí. Estas áreas se definirían como de "interacción" a partir de la ubicación que presentan las aberturas, abriéndose hacia ese espacio ciertamente compartido. La conformación espacial de estas áreas pareciera resultar de la disposición de las estructuras que las delimitan, o en realidad las encierran y envuelven. Al mismo tiempo, podemos considerar que la ubicación de estas estructuras pudo haber estado condicionada por la necesidad de este espacio abierto. Estas "áreas de interacción" permiten el relacionamiento con el interior de las estructuras a través de las aberturas, siendo que, de alguna manera, son parte de la circulación que permite recorrerlas. Si consideramos esto a la luz de los casos concretos podemos observar cómo en el Alero 1, las chullpas 02,03 y 04, por un lado, y las 06, 07, 08 y 09, por el otro, están vinculadas por su frente, con sendos espacios abiertos (Figura 16). En el Alero 3 también se reconoce esta situación, con al menos dos "áreas de inte- 


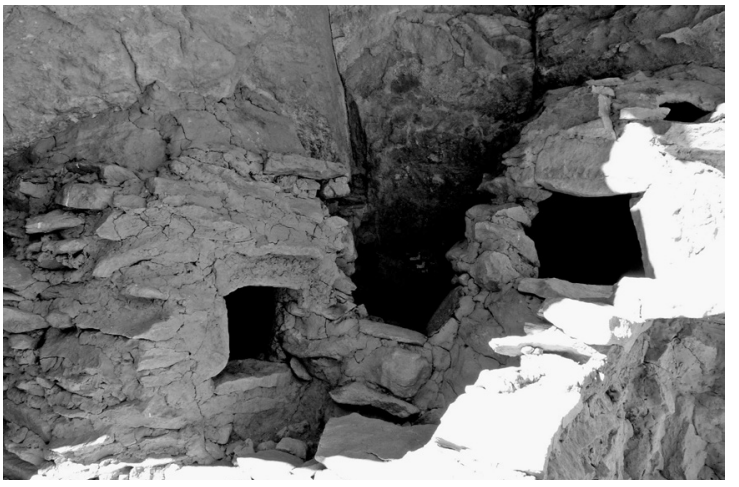

Figura 16. Una de las dos áreas de interacción propuesta para el Alero 1.

racción": una vinculando las estructuras 03, 05 y 06 (tal vez también 04) y otra, con 07 y o8. Finalmente, el caso del Alero 2 es interesante observar que la única "área de interacción" que se observa se encuentra delimitada no solo por las estructuras sino también por el muro frontal, que además condiciona el acceso a este espacio. A su vez, parte del arte rupestre participa en la caracterización y significación de esta "área de interacción".

La orientación de las aberturas. La cuestión de la orientación de las aberturas de las estructuras chullparias ha llamado la atención a distintos investigadores ocupados en la problemática, observando tanto alguna preferencia cardinal, especialmente hacia el este (Isbell 1997), como vinculaciones con ciertas topografías sacralizadas (Berenguer et al. 1984). En el caso de las estructuras que hemos analizado, el $64,2 \%$ de las aberturas presentan orientaciones que van del norte al este, mientras que el resto se reparte en los demás cuadrantes. Más allá de la aparente preferencia por el este y el norte, la incidencia de otras orientaciones es significativa y merece ser tomada en cuenta puesto que podría mostrar que no es el único factor de decisión a la hora de ubicar una abertura. Con los datos disponibles, es posible esbozar otra lógica además de la cardinal, basada en el interrelacionamiento de las estructuras, especialmente dentro de los agrupamientos tipo celulares (Figura 5).

Si nos detenemos en el Alero 1, de las nueve estructuras de las que tenemos datos, una se orienta al este, tres al norte, dos al noreste y tres al oeste. Esto nos permite observar cómo, bajo similares condiciones de emplaza-

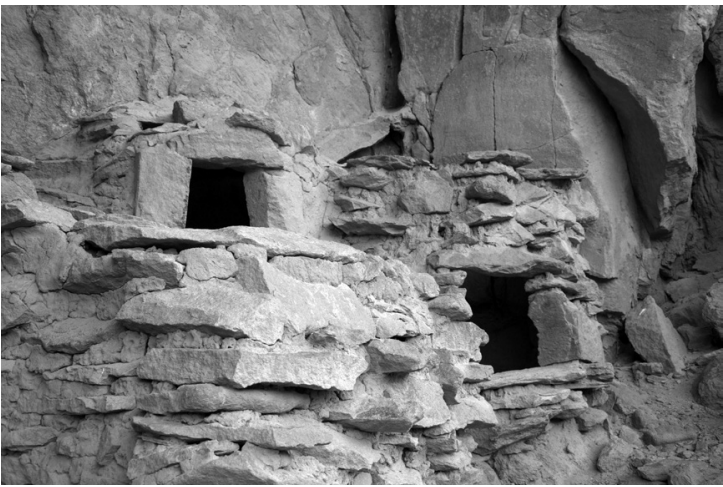

Figura 17. Situación donde se observa un desarrollo vertical en Alero 1. Nótese cómo la base pedestal eleva la estructura o 8 sobre el nivel de la o7 y la o9.

miento dentro de un alero, los vanos pueden tener orientaciones diferentes. Particularmente las aberturas de las estructuras 06, 08 y o9 del mismo agrupamiento se dispusieron de tal manera que se "observan" entre sí. La definición de las "áreas de interacción" permite comprender mejor este planteo, puesto que la disposición de una abertura pareciera surgir de la búsqueda de un vínculo con una u otra de estas áreas. Visto de otra manera, estos espacios se definen como tales a partir de la disposición de los vanos. Esta relación entre las aberturas puede ser pensada, como veremos, a partir de la ritualidad que se habría desarrollado en torno a estas estructuras, en términos colectivos.

Las áreas de circulación. Las "áreas de interacción" pueden ser pensadas también como aquellos espacios que permiten un recorrido por las distintas estructuras dentro de los agrupamientos. En el caso de las configuraciones alineadas esto se observa de una manera relativamente sencilla, puesto que se trata de un único espacio frontal continuo que permite tener una percepción, un reconocimiento, de cada una de las estructuras y acceder a, o relacionarse con, éstas. Para los aleros que venimos analizando, dada su condición celular, estos recorridos interiores adoptan formas más complejas y pueden ser analizados como espacios intermedios que vinculan las distintas "áreas de interacción" y, a su vez, las estructuras. En el Alero 1 se reconoce la existencia de un corredor en forma de U, por delante de las estructuras 05 y 07 , que articula las dos "áreas de interacción" propuestas. En el Alero 3, también es posible pensar en un recorrido por el frente del alero, que relaciona las dos "áreas de 
interacción" y a su vez permite alcanzar la estructura de doble abertura. Aunque estos corredores pueden definirse como espacios vacíos entre las estructuras, lo cierto es que parecieran estar concebidos como parte de la construcción del espacio de los aleros. En este sentido, como ya lo adelantamos, la posibilidad de acceder a todas las construcciones, tal vez dentro de un recorrido ceremonial ritual, es parte de la definición de un agrupamiento.

Los desarrollos verticales. La concepción de la espacialidad de los aleros no se da exclusivamente en planta, sino que implica un modelado tridimensional, con el aprovechamiento de distintos planos en altura. Si nos detenemos en el Alero 1, podemos diferenciar tres tipos de decisiones que hacen al desarrollo en altura. En primer lugar, en el sector izquierdo del alero se registra un escalón de unos 0,30 metros de altura, que salva el desnivel que presenta el piso hacia la parte posterior. Es importante considerar que este escalón participa en la disposición de las estructuras, elevando aquellas que se encuentran hacia atrás con respecto a las que se encuentran adelante. Incluso el plano del cerramiento lateral de la estructura O2, coincide con el plano de la alzada de este escalón. Un segundo tipo de decisiones constructivas está vinculado con las ya mencionadas bases pedestal, de entre 0,80 y 1 metro de altura, que elevan el desarrollo propiamente dicho de las estructuras 04, 06 y 08. El punto más alto de estas bases se ubica en el mismo nivel que el final del desarrollo de las estructuras que se encuentran delante, 05 y 07. Es decir, la existencia de estas tres bases pedestal permite que 04, 06 y 08, particularmente sus aberturas, sean visibles a pesar de la existencia de las otras dos estructuras adosadas. Finalmente, debemos considerar el aprovechamiento de la saliente rocosa, que conforma un segundo nivel, a tres metros del piso, donde se ubica la estructura 10, de doble abertura. Esta suma de decisiones termina resultando en lo que podríamos considerar cuatro niveles de construcciones: uno por debajo del escalón, otro inmediatamente encima de éste, el siguiente conformado por las bases pedestales y el último a partir de la saliente rocosa (Figura 17).

En el Alero 2, vuelven a presentarse dos de los elementos que acabamos de plantear. Por un lado, todo el interior se encuentra 0,30 metros por encima del piso exterior, siendo que ese desnivel está contenido por el muro de cerramiento, con un escalón, ubicado en el umbral, que salva esa diferencia. A partir de esto, las estructuras ubicadas afuera, o1 y 02 , se encuentran por debajo de $03 \mathrm{y}$ 04 en el interior. Con la estructura 05, de doble abertura, se presenta una situación similar a la del Alero 1 , al estar emplazada en una saliente rocosa que la eleva unos 1,5 metros por encima del piso del alero. En cuanto al Alero 3, podemos observar también una pendiente hacia afuera que genera que las distintas estructuras que conforman el agrupamiento se ubiquen en distintos niveles, sin que se observe la construcción de escalones. En este alero también la estructura de doble abertura (OI) se ubica distante del resto, en una posición más elevada, aunque en este caso se trata de solo 0,70 metros, producto de un escalón natural de la peña.

Este conjunto de decisiones, vinculadas con una definición tridimensional del espacio, nos aproxima a la concepción del agrupamiento como una totalidad que va más allá de una suma de estructuras. La situación referida para el Alero 1 nos permite pensar en una voluntad de dar visibilidad a todas las construcciones, de tal manera que es posible lograr una lectura integral del conjunto reconociendo cada una de las chullpas y, entonces, de los ancestros.

Los cerramientos frontales. Tanto en el Alero 1 como en el 2, hemos registrado un muro frontal de cerramiento que delimita, envuelve y contribuye a construir una interioridad. En el primer caso, este muro de cerramiento, que en gran parte se encuentra derrumbado, pareciera haber tenido un trazado más o menos recto cubriendo la totalidad del frente, e incluyendo lo que actualmente se conserva de la parte frontal de la estructura $\mathrm{Ol}^{7}$. En el Alero 2, el muro se presenta en mejores condiciones de conservación, tiene una forma rectangular y se proyecta levemente por fuera del alero, siendo que en el lado norte se ubica la abertura.

Si bien hemos registrado solo en estos dos aleros la exis-

7 Vale recordar que esta estructura sólo conserva su muro frontal con la abertura, por lo que eventualmente en algún momento pudo haber sido desarmada y solo permanecer como parte del cerramiento del alero. En una situación límite, cabe la posibilidad de que no haya sido una estructura chullparia y la abertura que se observa haya sido directamente la del muro, es decir, la del alero. 
tencia de muros de cerramiento, hay una serie de puntos significativos para analizar. Estos muros con sus aberturas nos habilitan a pensar en la existencia de un cierto recorrido prescriptivo. Al menos en el caso del Alero 2, era posible ingresar al agrupamiento por un sector prefijado que, por otra parte, se estableció de acuerdo a las dataciones (ver supra) antes de la elevación de las estructuras del interior del alero. Debemos observar que este muro restringe y condiciona las miradas desde el exterior, y a su vez la abertura dirige la percepción hacia un cierto punto de vista de la interioridad. Si nos detenemos en la morfología de este cerramiento es significativo que haya sido levantado en tres tramos y que la abertura esté en uno de los que podríamos considerar laterales. Esta ubicación condiciona que al momento de acceder al alero se observe el frente de las tres estructuras, particularmente la superior (05). Ciertamente esto no hubiera ocurrido de la misma manera si el ingreso se hubiera dado por el tramo central del cerramiento. Es en este sentido que nos referimos a la prescripción de un determinado recorrido tanto en sentido literal como visual (ver Figura 9).

Estos muros generan una envolvente que contiene a la totalidad de las estructuras dentro de un ámbito cerrado en el interior de los aleros. De esta manera, existe un cierto paralelo entre la abertura de la estructura chullparia y la abertura del alero. En la relación cerramiento abertura se habilita o restringe, en forma controlada, tanto la entrada como la salida de las distintas entidades. Si esto fuera así, estaríamos ante una sucesión de coberturas, de envolventes. Primero con la estructura chullparia y su tratamiento especial en su interior, luego el alero con su cerramiento $y$, tal vez, la ubicación de estos aleros en quebradas más o menos angostas, que también envuelven y protegen ( $\mathrm{Ri}$ vet 2014a).

\section{Un proyecto en el tiempo}

En cierto modo, cada una de estas estructuras chullparias puede ser pensada como una totalidad, con una existencia autónoma ${ }^{8}$. Lo cierto es que el enfrentarnos al estudio de los agrupamientos nos obliga a pensarlas en términos colectivos. La construcción de una nueva

8 De hecho, nos hemos dedicado al análisis de las estructuras en sí mismas en un trabajo anterior (Rivet 2014). estructura que se adosa, compartiendo su muro, a una preexistente implica un cambio en la entidad de la primera, puesto que sus condiciones de existencia ya no son las mismas. La incorporación de esta nueva construcción provoca cambios concretos en la materialidad de la primera, incluyendo operaciones que van desde el "alisamiento" de un muro lateral exterior, al devenir en interior, hasta las soluciones involucradas en la definición de los frentes, que pueden terminar siendo continuos. Al pensar estas estructuras debemos considerar que cada una es una entidad en sí misma, pero que a su vez, son una parte de entidades mayores que las integran, es decir, los agrupamientos. Por otra parte, el hecho que la estructura que se incorpora no tenga un muro "propio" continuo, sino que recurra parcialmente al preexistente, nos puede llevar a plantear que no hay una relación absolutamente simétrica entre las estructuras, sino que más bien, se presenta una cierta dependencia de una hacia la otra. De alguna manera, la primera que se elevó sostiene, literalmente, a las que se irán sumando en el tiempo.

Tal como lo hemos desarrollado, las estructuras no solo se integran, sino que también se diferencian de otras, formando, en un mismo agrupamiento, distintas "líneas de crecimiento". Es decir, por alguna razón, ante la construcción de una estructura se tomó la decisión de vincularla con una de las existentes y no con otras. Como lo propusimos, las estructuras se disponen de tal manera que terminan resultando determinadas "áreas de interacción" que pueden interpretarse dentro de un determinado "proyecto" de conformación del agrupamiento y del espacio chullpario, sostenido a lo largo del tiempo. La entidad de estos agrupamientos es entonces, por definición, dinámica y habría estado sujeta a constantes cambios, dentro de lo que podríamos considerar un patrón estable. Es así como la construcción sucesiva no se presenta como aleatoria y preferimos hablar de un "proyecto", que implicaría la comprensión de ciertos códigos espaciales y arquitectónicos que son "leídos", “decodificados", a la hora de elegir un lugar para construir una nueva estructura.

Al referirnos a este "proyecto" debemos realizar algunas consideraciones. No estamos proponiendo que haya existido una determinada conformación a priori, a modo de un plano que luego se respeta. En este senti- 
do, las dos "áreas de interacción" que hemos propuesto para el Alero i seguramente no estaban contempladas por quienes comenzaron a intervenir ese espacio. Debemos pensar, más bien, en un proceso de diseño que se da en la práctica de construir y de vivir esos espacios a lo largo del tiempo, de alguna manera guiado por determinadas normas compartidas que forman parte del habitus (Bourdieu 2007). Estas normas están sujetas a cambios en el tiempo sin que esto implique por ello una ruptura con las condiciones de existencia en que se generaron. Ante la situación de sumar una nueva estructura, quienes la construirían tenían mayores o menores márgenes de acción para decidir dónde la emplazarían, en relación con qué otra chullpa, qué morfología tendría, etc. En el marco de estos agrupamientos se ponen en juego, entonces, tanto las subjetividades, en términos personales o grupales, como las normatividades que se producen, reproducen y modifican colectivamente en el tiempo.

Antes de proseguir con el texto, vale la pena que nos detengamos brevemente en la referida presencia de arte rupestre en dos de los aleros (Caso 1 y Caso 2), puesto que hace a la concepción en el tiempo de estos espacios. Sin ahondar en las características específicas de este arte, que están siendo desarrolladas en otro artículo (Rivet 2014b), si podemos observar que los distintos conjuntos plásticos se presentan en su interacción con las estructuras chullparias bajo tres modalidades: sobre las paredes rocosas del alero por fuera de las estructuras, sobre las paredes rocosas del alero en el interior de las construcciones y en el interior o exterior de los mismos muros de las estructuras. Particularmente el segundo de estos casos, se vuelve especialmente significativo puesto que dada la ubicación de estos conjuntos debieron haber sido pintados antes, o al mismo tiempo, de la elevación de la estructura. En la primera situación, las estructuras chullparias se sumaron a un espacio que ya estaba semantizado, y los sentidos asociados a ese arte habrían sido reapropiados o amplificados a través de la conformación del agrupamiento. Es interesante pensar en cómo dos registros (el plástico y el arquitectónico) están operando en un mismo espacio. La continuidad en el análisis del arte rupestre nos podrá mostrar cómo los motivos presentes se podrían relacionar con lo que aquí proponemos sobre los agrupamientos.

\section{* Ancestros y parentesco}

\section{La ancestralidad en los Andes y el lugar de las chullpas}

A comienzos de la década de 1970, Zuidema (1973) planteó que el "sistema ancestral" era una clave esencial para la comprensión de la organización política, económica y social en los Andes. A partir de allí, numerosos investigadores han enfatizado este rol central de los ancestros en la definición de las sociedades andinas. Lau, en particular, subrayó que las ceremonias destinadas a éstos "funcionaban como instituciones socio-religiosas vitales para el Imperio Inka tanto como para las sociedades de pequeña escala durante la prehistoria Andina tardía, y persisten hoy en día como actividades importantes para los grupos tradicionales en las tierras altas" (2002: 279). Tanto las referencias etnográficas como las etnohistóricas han mostrado que los ancestros son agentes activos en la consolidación de los lazos dentro de los grupos sociales y la legitimación de los vínculos con sus territorios, constituyéndose entonces como fuentes de autoridad (Lau 2008). A través de fuentes etnohistóricas, Zuidema (1973) planteó que la referencia a un ancestro podía estar asociada con la defensa de los derechos sobre la tierra a través del reconocimiento de la línea de descendencia por medio de la cual ésta fue heredada. Es decir, en la veneración de un determinado ancestro se habría estado afirmando la relación con aquél, y por lo tanto, los derechos sobre los recursos que habilitaba. En los términos de Nielsen, dentro de los grupos prehispánicos tardíos, "los recursos y el rango que usufructuaba cada linaje o ayllu eran los que les correspondían como descendientes de determinadas figuras ancestrales, reales o míticas" (2006a: 127). Este mismo investigador señaló que durante el período de Desarrollos Regionales el carácter corporativo y descentralizado de las formaciones políticas en los Andes se fundaba en el culto a los ancestros, puesto que el rol de la autoridad dentro del grupo era "contingente", surgiendo precisamente de la vinculación con éstos (Nielsen 2006c). A los efectos de este artículo, lo que nos interesa reforzar es que hablar de ancestros implica tratar acerca de las formas de organización social y de relaciones de parentesco a través de las cuales se interceptan los vínculos de aquellos con las personas. Como veremos, la materialidad tiene un rol superlativo para la construcción y afianzamiento de estos lazos. 
Debemos considerar el carácter dinámico y flexible propio de la ancestralidad en los Andes. En este sentido, el "orden ancestral" no era inalterable, sino que tuvo la capacidad de modificarse a lo largo del tiempo en virtud de los cambios en los contextos sociales y políticos (Gose 2008). A través de la veneración a un ancestro, performativamente, se habrían establecido las pertenencias a un determinado grupo, sin que esto implicara necesariamente una relación genealógica estricta. Es así como una alianza entre dos grupos podía sellarse a través del reconocimiento de un ancestro en común (Gose 2008). Esto implicaba a su vez relaciones entre los ancestros y una determinada jerarquía como parte de un colectivo (Lau 2008) dentro de lo que podríamos considerar una comunidad de antepasados.

Diferentes crónicas coloniales llamaron la atención sobre la intensidad de las celebraciones rituales que las poblaciones realizaban a favor de sus ancestros y antepasados (Arriaga 1621; Cobo 1893 [1653]; Cieza 1922 [1553]; Guamán Poma 1992 [1615]; Álvarez 1998 [1588], entre otros). Esto estaba asociado con la existencia de relaciones cercanas de las personas con ellos, que conllevaban a su vez el brindarles ciertas ofrendas en determinados momentos. Algunos autores han asociado estas acciones con un cierto acuerdo de reciprocidad para la obtención de los favores de los antepasados, a cambio de su cuidado y memoria (Lau 2008). Como ha sido ilustrado en diferentes trabajos arqueológicos (p.e. Aldunate y Castro 1981; Isbell 1997; Gil García 2010) las estructuras chullparias eran uno de los puntos de encuentro periódicos donde se establecían dichas relaciones entre personas y antepasados, a través de las acciones rituales.

La materialidad de los ancestros. En un trabajo pionero por su enfoque, Duviols propuso que las piedras huancas eran la "litificación de los ancestros", tal que el huanca era entendido como "el equivalente del ancestro ejemplar del ayllu, ancestro que fundó la marca o la chacra [...] y que continúa velando por ellos" (1979: 10, traducción propia). Estos huancas se emplazaban en lugares significativos de acuerdo a la historia del ancestro y, a su vez, sus relaciones de parentesco, jerarquías y prestigios habrían sido equivalentes a aquellas que existían entre los grupos humanos, tal que estudiar los huancas sería estudiar a los ancestros (Duviols 1979). Más allá del caso puntual de estos monolitos, los ancestros se habrían materializado a lo largo del tiempo en distintos objetos, e incluso el arte rupestre, a través de diferentes procesos de transformación (Galdames 1987; Aschero y Korstanje 1996; Kaulicke 2001, entre otros), situación que ha sido observada también en relación con las estructuras chullparias (Isbell 1997; Nielsen 2008). El planteo general sería que, de alguna manera, la lógica que subyace en las relaciones entre las personas vivas, entre los ancestros y entre las estructuras chullparias son simétricas, en términos de Latour (2008). Es decir, estas chullpas, no tienen un papel subsidiario, menor o lateral, en la vida social de las personas y los ancestros, sino que son activos partícipes en las negociaciones, disputas, rupturas, continuidades y establecimiento de los vínculos sociales. Nielsen (2010), en lo que es un punto de partida interpretativo para nosotros, ha sido incluso más explícito respecto a la condición de las chullpas al entender que éstas eran "encarnaciones de los propios antepasados, capaces de hacer lo que los antepasados hacían" (2010: 339). Si avanzamos aún más, debemos comprender que la chullpa no se construye a posteriori del acto de erigir a un ancestro, sino que en la construcción misma de esta estructura es que esto ocurre. Al hablar de la construcción, nos referimos literalmente a los procedimientos, elecciones y gestos técnicos a partir de los cuales, performativamente, se "crearía" un ancestro.

Relaciones entre chullpas, ancestros y personas. La construcción a lo largo del tiempo de los agrupamientos es indisociable de una ritualidad más o menos continua sobre esos espacios. Esto pudo haber involucrado incluso a las operaciones de mantenimiento constructivo sobre las estructuras ${ }^{9}$, tal vez, como parte de las relaciones con los ancestros, que habrían sido estrechas y continuas en el tiempo. Con respecto a esto, Lau sostiene que: "En tanto la veneración de los ancestros está generalmente en la esfera de los grupos de parentesco, existe a menudo una relación íntima entre las imágenes de culto (ancestros) y sus hacedores [makers] y sus usuarios/manipuladores (descendientes). En efecto, los documentos coloniales sugieren que las efigies, especialmente los fardos fune-

9 Consideramos como operaciones de mantenimiento a aquellas que se registran en diferentes estructuras, a posteriori de haber sido erigidas. Nos referimos a intervenciones recurrentes tales como reparaciones de techos y muros o adiciones puntuales de barro para mejorar el anclaje con la pared rocosa. 
rarios [mummy bundles], eran tratados esencialmente como miembros estimados de la familia." (2008: 1041, traducción propia).

En efecto, se ha observado que existía, y de hecho existe, una profunda interacción entre las personas y sus ancestros y antepasados dentro de las sociedades andinas. Éstos tenían, y siguen teniendo, un papel activo en la conformación de los grupos sociales y en el sostenimiento de sus derechos, y una notable capacidad de agencia para incidir en la vida de las personas. Es en este sentido que Isbell (1997) propuso que el ayllu era la historia de los ancestros y que, a su vez, la historia de éstos era la historia de sus chullpas. Al respecto, Salomon, a partir de su trabajo con fuentes históricas del siglo XVIII para el área Arequipa, observó que el culto a las momias implicaba relaciones que involucraban a los grupos domésticos. Es así como "Los viernes por la noche, cada grupo familiar iba por su cuenta hasta el santuario que representaba a la "familia" a través de sus ancestros (...) Por ello, debemos presumir que cada santuario de ancestros representaba a varios hogares que componían la unidad llamada "familia" (...) La "familia", representada por el santuario, era concebida como una institución permanente" (Salomon 1990: 160).

Si espacializamos la indagación sobre este tipo de relaciones, es estimulante observar que Zuidema (1973, en Lau 2008) sugirió un paralelo entre la posición física de las efigies y las distintas líneas de descendencia. En la misma línea, el análisis que venimos proponiendo sobre los agrupamientos nos permitiría al menos preguntarnos sobre ciertas articulaciones que podrían existir entre las configuraciones espaciales de éstos y las conformaciones sociales. Cabría pensar, entonces, si lo que hemos definido como "líneas de crecimiento" dentro de los agrupamientos, no se correspondería de la misma manera con distintas líneas de descendencia que se desprendieron en el tiempo de un ancestro "fundador" compartido, mítico o real. Si esto fuera así, una observación general sobre el agrupamiento nos expondría ante distintos grupos emparentados entre sí. La incorporación de una nueva estructura a una u otra "línea de crecimiento" podría estar respondiendo a la pertenencia de ese ancestro, dentro de las tramas del parentesco. Lo que se estaría formando en el agrupamiento, entonces, serían "comunidades de ancestros" emparentados entre sí, y una expresión material explícita de las líneas de descendencia, en una suerte de "mapa de relaciones sociales". Podemos ir más allá de la idea de "la expresión" para considerar en realidad que en estos agrupamientos se están construyendo, reforzando y actualizando determinados vínculos, en pos del reconocimiento de la posición de las personas dentro de la sociedad y el establecimiento de sus derechos sobre sus territorios.

En el análisis constructivo planteamos que al adosar una nueva estructura observamos que aquella que se incorpora se apoya, literalmente en términos estructurales, sobre la preexistente. De esta manera es dependiente de aquella, puesto que no tiene una caja muraria completamente autónoma. Si vemos esto a la luz de las relaciones sociales, no deja de ser interesante pensar si el ancestro que se añade a una línea de descendencia no requiere de un vínculo con las entidades preexistentes para afirmar su propia condición. Visto desde la totalidad de un agrupamiento, por ejemplo el del Alero 1, nos encontraríamos con una suma de ancestros que sostienen su autoridad como tales a partir de su vínculo físico con los precedentes. Si seguimos las "líneas de crecimiento" propuestas, llegaríamos sólo a una o dos estructuras primigenias, que podrían pensarse, por qué no, en términos de ancestros fundadores. Por otra parte, el encadenamiento de estructuras con nuevas construcciones que se suman en el tiempo, se vuelve también significativo en un contexto en el que el sostenimiento del culto a los ancestros encarnaba la continuidad de las líneas de descendencia (Salomon 1990).

¿Ritualidades en las "áreas de interacción"? Existen múltiples referencias etnohistóricas a los densos rituales que se han desarrollado a propósito de los ancestros por parte de las sociedades andinas. Las crónicas en particular muestran cómo los sepulcros eran uno de los principales escenarios para el desarrollo de estas acciones. Es así como Arriaga observó: "héchanles muy disimuladamente chicha en el sepultura, para que bevan, y muy al descubierto ponen cuando les hazen las honras, comidas cocidas, y asadas sobre la sepultura, para que comen" (Arriaga 1621: 35).

En un sentido similar, Cobo refirió: "Celebraban sus aniversarios acudiendo á ciertos tiempos á las sepulturas, y abriéndolas, renovaban la ropa y comida que en ellas habían puesto, y ofrecían algunos sacrificios" (Cobo 1893 [1653]: 238). 
No podemos dejar de observar que este tipo de referencias están presentes a su vez en distintas etnografías sobre las sociedades actuales en los Andes en las que se da cuenta de los diferentes rituales anuales que se llevan a cabo en relación con el "culto y la celebración a los muertos", con las distintas ofrendas ("turcos") que se les brindan frente a las sepulturas o en las mesas rituales (Harris 1983; Fernández Juárez 2001; Kessel 2001; Vilca 2009; Rivet y Tomasi 2013, entre otros). En el marco de estas acciones se establecen, y regulan, las relaciones entre las personas y los difuntos dentro de vínculos que, como ha sido propuesto, están basados en la reciprocidad en la que los descendientes proveen de cuidados a sus ancestros y éstos les otorgan sus favores, particularmente en torno a la fertilidad y la reproducción.

Desde la arqueología, en Toconce (norte de Chile), Aldunate y Castro (1981) excavaron frente al umbral de distintas chullpas encontrando uno o más fogones en cada una, con huesos de animales quemados, restos de maíz y fragmentos cerámicos entre las cenizas. A partir de esto, y de información etnohistórica, interpretaron que sería el resultado de "una suerte de banquete ritual celebrado en el exterior de las estructuras, culminado con ofrendas consumidas por el fuego para hacerlas llegar a los antepasados" (1981: 154). A esto se sumaría el hallazgo de malaquita y conchas marinas dentro de los mismos fogones, que reforzaría la situación ritual de la quema. Como hemos ya indicado, no hemos realizado al momento excavaciones en los agrupamientos chullparios que nos permitan reconocer una situación similar. Sin embargo, a la luz de las referencias citadas, no podemos dejar de preguntarnos sobre las potenciales situaciones de comensalidad que podrían haberse desarrollado en los espacios que hemos definido como "áreas de interacción". Si fuera el caso, en tanto las aberturas de distintas estructuras se abren sobre una misma área, tal vez deberíamos pensar en acciones rituales que podrían haber tenido una connotación colectiva con el culto a distintos ancestros en un mismo momento, o en realidad a un colectivo de ancestros como una unidad. Si volvemos sobre la existencia de muros de cerramiento en los Aleros 1 y 2, debemos retomar la idea de que en cierta manera estos aleros se constituyen como una única gran estructura chullparia que integra a distintos ancestros vinculados entre sí. Por otra parte, en estas eventuales celebraciones colectivas no sólo se habrían reforzado las relaciones con los an- cestros, sino también entre las distintas personas que participaban en estas prácticas. De esta manera, en torno a los agrupamientos, y especialmente de las "áreas de interacción", se habrían presentado tres esferas de vinculación: entre los ancestros y sus descendientes, entre los ancestros mismos y entre las personas (Chacama 2003).

\section{* Consideraciones finales}

A lo largo de este trabajo nos hemos preguntado acerca de la posibilidad de que en torno a los agrupamientos chullparios se hubieran establecido vínculos cercanos entre personas y ancestros, y entre estos últimos. Esto se observaría a través de las interacciones entre las mismas estructuras chullparias, donde se explicitarían materialmente estas relaciones, a partir de lo que hemos denominado "líneas de crecimiento" y "áreas de interacción". Estas "líneas de crecimiento" pondrían en evidencia la interdependencia dentro de un colectivo de ancestros, emparentados entre sí. En este sentido, mientras que ciertas estructuras aparecen con todo su perímetro completo y se habrían constituido como "puntos de partida" en los agrupamientos, las subsiguientes se adosaron a las anteriores de tal manera que necesitaban de éstas, estructuralmente, para sostenerse. Aunque la lectura puede ser un tanto literal, no podemos dejar de pensar en que la condición de este nuevo ancestro como tal se sustentaba en sus relaciones con los anteriores.

Al hablar de "líneas de crecimiento", en plural, hemos intentado mostrar que no se trataba de un único encadenamiento sucesivo sino, más bien, de distintas líneas, que pudieron haber reconocido un ancestro compartido. Si retomamos la perspectiva de Gose (2008), y también de Nielsen (2006a y b), debemos considerar las potenciales negociaciones que se habrían estado estableciendo entre grupos a través de estas estructuras. De esta manera, tal vez, al emplazar una estructura en una determinada línea de crecimiento con relación a otra se podrían haber constituido vínculos entre grupos distintos. En cualquier caso, esta construcción de una nueva estructura implicaba una negociación que conllevaba un cambio en las condiciones de la preexistente. Estas consideraciones sobre las líneas de crecimiento nos llevan a volver sobre las "áreas de interacción" dentro de los agrupamientos. Tal como lo planteamos, éstas se constituyen como espacios abiertos que vinculan distintas estructuras de di- 
ferentes modos, especialmente a través de las aberturas. Es interesante considerar que cualquier tipo de práctica que se realizara allí, aunque se orientara a un ancestro en particular, implicaba la presencia de otros. Especialmente la conformación en los aleros nos ha mostrado la posibilidad de distintas áreas de interacción en un mismo agrupamiento. Cabe preguntarnos si no nos estamos enfrentando a distintos niveles de agregación social sucesivos en los que se ponían en juego y se activaban distintos vínculos entre las personas.

\section{* Referencias Citadas}

ALBECK, M.E. y M. RUIZ. 2003. El Tardío en la puna de Jujuy: poblados, etnias y territorios. Cuadernos de la Facultad de Humanidadesy Ciencias Sociales, Universidad Nacional de Jujuy 20: 199-221.

ALDUNATE, C. y V. CASTRO. 1981. Las chullpa de Toconce y su relación con el poblamiento altiplánico en el Loa Superior. Período Tardío. Tesis para optar al grado de Licenciado en Filosofía con Mención en Prehistoria y Arqueología. Universidad de Chile (Ms).

ASCHERO, C. y A. KORSTANJE. 1996. Sobre figuraciones humanas, producción y símbolos. Aspectos del Arte Rupestre del Noroeste Argentino. En XXV Aniversario Museo Arqueológico Dr. Eduardo Casanova, M. C. Rivolta (Ed.), pp. 13-32. Instituto Interdisciplinario Tilcara, Facultad de Filosofía y Letras, Universidad de Buenos Aires, Tilcara, Jujuy.

ARRIAGA, P. J. 1621. Extirpacion de la Idolatria del Piru. Gerónimo de Contreras, Impresor de Libros, Lima.

BERENGUER, J., ALDUNATE, C. y V. CASTRO. 1982. Orientación orográfica de las chullpas en Likán: la importancia de los cerros en la fase Toconce. En Simposio Culturas Atacameñas. XLIV Congreso Internacional de Americanistas, B. Bittman (Ed.), pp. 175220. Universidad del Norte, Antofagasta.

BOURDIEU, P. 2007 [1980]. El sentido práctico. Siglo XXI Editores, Buenos Aires.

BRONK RAMSEY, CH. 2009. Bayesian analysis of radiocarbon dates. Radiocarbon 51 (1): 337-360.

CABRERA, A. 1953. Esquema fitogeográfico de la República Argentina. Revista del Museo, Universidad Nacional de Eva Perón, Botánica 8 (33): 87-168.

CASANOVA, E. 1938. Investigaciones arqueológicas en Sorcuyo. Puna de Jujuy. Anales del Museo Argentino de Ciencias Naturales, Serie Antropología, Etnología y Arqueología 39: 423-456.

CHACAMA, J. 2003. Identidad espiritual y organización social en los Andes centrales. Revista Historia Indígena 7: 139-158.
CIEZA DE LEON, P. 1922 [1553]. La crónica del Perú. Editorial Calpe, Madrid.

COBO, B. 1893 [1653]. Historia del Nuevo Mundo. Sociedad de Bibliófilos Andaluces, Sevilla.

DEBENEDETTI, S. 1930. Chulpas en las cavernas del Río San Juan Mayo. Notas del Museo Etnográfico 1: 5-50.

DIETLER. M. y I. HERBICH. 1998. Habitus, Techniques, Style: An Integrated Approach to the Social Understanding of Material Culture and Boundaries. En The Archaeology of Social Boundaries, M. Stark (Ed.), pp. 232-263. Smithsonian Institution Press. Washington, D.C.

DUCHESNE, F. y J. CHACAMA. 2012. Torres funerarias prehispánicas de los Andes Centro-Sur: muerte, ocupación del espacio y organización social. Estudio comparativo: Coporaque, Cañón del Colca (Perú), Chapiquiña, precordillera de Arica (Chile). Chungara. Revista de antropología chilena 44 (4): 605-619.

DUVIOLS, P. 1979. Un simbolisme de l'ocupation, de l'amagement et de l'explotation de l'espace. Le Monolithe Huanca et sa fonction dans les andes Prehispaniques. L'Homme XIX (2): 7-31.

FERNÁNDEZ JUÁREZ, G. 2001. Almas y difuntos: ritos mortuorios entre los aymara lacustres del Titicaca. Chungara. Revista de antropología chilena 33 (2): 201-219.

GALDAMES ROSAS, L.A. 1987. Vitalidad de la Piedra y Petrificación de la Vida: notas sobre Mentalidad Andina. Diálogo Andino 6:128-143.

GIL GARCÍA, F.M. 2010. Dentro y fuera parando en el umbral: construyendo la monumentalidad chullparia. Elementos de tensión espacial para una arqueología del culto a los antepasados en el altiplano andino. Diálogo Andino 35: 25-46.

GISBERT, T. 1994. El señorío de los Carangas y los chullpares del Río Lauca. Revista Andina 12: 427-485. 
GOSE, P. 2008. Invaders as ancestors: On the intercultural making and unmaking of Spanish Colonialism in the Andes. University of Toronto Press, Toronto.

GUAMAN POMA DE AYALA, F. 1992 [1615?]. El primer nueva corónica y buen gobierno. Siglo Veintiuno Editores, México, D.F.

HARRIS, O. 1983. Los muertos y los diablos entre los laymi de Bolivia. Chungara 11: 135-152.

ISBELL, W. H. 1997. Mummies and mortuary monuments. A postprocessual prehistory of central Andean social organization. University of Texas Press, Austin.

KAULICKE, P. 2001. Vivir con los ancestros en el Antiguo Perú. En La memoria de los ancestros, L. Millones y W. Kapsoli (Comps.), pp. 25-61. Editorial Universitaria, Universidad Ricardo Palma, Lima.

KESSEL, J.V. 2001. El ritual mortuorio de los aymara de Tarapacá como vivencia y crianza de la vida. Chungara. Revista de antropología chilena 33 (2): 221-234.

KRAPOVICKAS, P. 1958-59. Arqueología de la Puna Argentina. Anales de Arqueología y Etnología XIV-XV: 53-113.

KRAPOVICKAS, P. y E. CIGLIANO. 1962. Investigaciones arqueológicas en el valle del Río Grande de San Juan (Puna Argentina). Anales de Arqueología y Etnología XVII-XVIII: 71-118.

LATOUR, B. 2008. Reensamblar lo social. Una introducción a la teoría del actor-red. Manantial, Buenos Aires.

LAU, G. 2002. Feasting and ancestor veneration at Chinchawas, north highlands of Ancash, Peru. Latin American Antiquity 13 (3): 279-304.

2008. Ancestor images in the Andes. En The handbook of south american archaeology, H. Silverman y W. Isbell (Eds), pp. 10271045. Springer, New York.

LEMONNIER, P. 1992. Elements for an Anthropology of Technology. Anthropological Paper, Museum of Anthropology, University of Michigan, Ann Arbor, Michigan.

MILLER, D. 2005. Materiality: an introduction. En Materiality, D. Miller (Ed), pp. 1-50. Duke University Press, Chapel Hill.

MOLINIÉ, A. 1997. Buscando una historicidad andina: una propuesta antropológica y una memoria hecha rito. En Arqueología, antropología e historia en los Andes: homenaje a María Rostworowski, R. Varón Gabai y J. Flores Espinosa (Eds.), pp. 691-708. Instituto de Estudios Peruanos, Banco Central de Reserva del Peú, Lima.
NIELSEN, A. E. 1995. Architectural Performance and the Reproduction of Social Power. En Expanding Archaeology, J. Skibo, W. Walker y A.E. Nielsen (Eds.), pp. 47-66. University of Utah Press, Salt Lake City.

2006a. Pobres Jefes: Aspectos corporativos en las formaciones sociales pre-inkaicas de los Andes circumpuneños. En Contra la tiranía tipológica en arqueología: Una visión desde Suramérica, C. Gnecco y L. Langebaek (Eds), pp.121-150. Universidad de Los Andes, Ediciones Uniandes, Bogotá.

2006b. Plazas para los antepasados: Descentralización y poder corporativo en las formaciones políticas preincaicas de los Andes circumpuneños. Estudios Atacameños 31: 63-89.

2008. The Materiality of Ancestors. Chullpas and Social Memory in the Late Prehispanic History of the South Andes. En Memory Work: Archaeologies of Material Practices, B. Mills. \& W. H. Walker (Eds.), pp. 207-231. School of American Research Press, Santa Fe.

2010. Las chullpas son ancestros: Paisaje y memoria en el altiplano sur andino (Potosí, Bolivia). En El Hábitat Prehispánico. Arqueología de la Arquitectura y de la Construcción del Espacio Organizado, M. E. Albeck, C. Scattolin \& A. Korstanje (Eds.), pp. 329349. Facultad de Humanidades y Ciencias Sociales, Universidad Nacional de Jujuy, San Salvador de Jujuy.

RIVET, M.C. 2013a. Cruces e iglesias en un contexto chullpario: Arte rupestre colonial en las tierras altas atacameñas. Nuevo Mundo Mundos Nuevos [online] http://nuevomundo.revues. org/64960> ISSN 1626-0252

2013b. Estructuras chullparias, agencias y negociación de sentidos en Agua Delgada (Coranzuli, provincia de Jujuy), entre el Período Tardío y el Colonial. Tesis para optar al grado de Doctor en Arqueología. Facultad de Filosofía y Letras, Universidad de Buenos Aires.

2014a. La textura de los ancestros. Reflexiones en torno a las lógicas y sentidos de las estructuras chullparias (Coranzulí, provincia de Jujuy, Argentina). Boletín del Museo Chileno de Arte Precolombino 20 (en prensa).

2014b. Arte en contextos chullparios. Primera aproximación a las manifestaciones rupestres de Coranzuli (Jujuy, Argentina) (Ms).

RIVET, M.C. y J. TOMASI. 2013. Casitas y casas mochas: Los antiguos y los abuelos en sus arquitecturas (Coranzulí y Susques, provincia de Jujuy, Argentina). En Wak'as, diablos y muertos: alteridades significantes en el mundo andino, L. Bugallo y M. Vilca (Comps.). Ediciones de la Universidad Nacional de Jujuy, Instituto Francés de Estudios Andinos (IFEA) (en prensa). 
SALOMON, F. 1990. Culto a los ancestros y resistencia frente al Estado en Arequipa entre los años 1748 y 1754. En Resistencia Rebelión y Conciencia Campesina en los Andes siglos XVIII al XX, S. Stern (Ed.), pp. 148-163. Instituto de Estudios Peruanos (IEP), Lima.

SILLAR, B. 1996. The Dead and the Drying: Techniques for Transforming People and Things in the Andes. Journal of Material Culture 1 (3): 259-289.

SOJA, E. 1971. The political organization of space. Resource Papers, Association of American Geographers, Washington.

TANTALEÁN, H. 2006. Regresar para construir: Prácticas funerarias e ideología(s) durante la ocupación inka en Cutimbo, PunoPerú. Chungara. Revista de antropología chilena 38 (1): 129-143.
TABOADA, C. 2005. Propuesta metodológica para el análisis diacrónico de arquitectura prehispánica y la asignación de significado conductual discriminado. Aplicación en el Noroeste argentino. Anales del Museo de América 13: 139-172.

VIGNATI, M.A. 1938. Novissima Veterum. Hallazgos en la Puna jujeña. Revista del Museo de La Plata I, Sección Antropología 5: 53-91.

VILCA, M. 2009. Los ojos a la espera del sol maduro. La celebración de las almas en Llamerías, Puna de Jujuy. Estudios de Filosofía Práctica e Historia de las Ideas 11 (1): 45-51.

ZUIDEMA, T. 1973. Kinship and ancestorcult in three peruvian communities. Hernandez Principe's account of 1622. Bulletin de l'Institut Français d'Études Andines 2 (1):16-33. 